\title{
MULTIPLE INTERIOR LAYERS OF SOLUTIONS TO PERTURBED ELLIPTIC SINE-GORDON EQUATION ON AN INTERVAL
}

\author{
Tetsutaro Shibata
}

\begin{abstract}
We consider the perturbed elliptic Sine-Gordon ODE with two positive parameters $\mu$ and $\lambda$, and show the existence of solutions which have $2 n$ multiple interior layers for $\lambda \gg 1$. We also determine the location of multiple interior layers as $\lambda \rightarrow \infty$.
\end{abstract}

\section{Introduction and results}

We consider the perturbed elliptic Sine-Gordon equation on an interval

$$
\begin{aligned}
-u^{\prime \prime}(t)+\lambda \sin u(t) & =\mu f(u(t)), \quad u(t)>0, t \in I:=(-T, T), \\
u( \pm T) & =0,
\end{aligned}
$$

where $\lambda, \mu>0$ are parameters and $T>0$ is a constant. We assume the following conditions (A.1)-(A.4):

(A.1) $f$ is locally Lipschitz continuous, odd in $u$. Furthermore, $f(u)>0$ for $u>0$.

(A.2) There exist constants $C>0$ and $p>1$ such that $|f(u)| \leq C\left(1+|u|^{p}\right)$ for $u \in \mathbb{R}$.

(A.3) $f(u) \leq C u$ for $0<u \ll 1$, where $C>0$ is a constant.

2000 Mathematics Subject Classification. 35Q53, 34B10.

Key words and phrases. Multiple interior layers, Sine-Gordon, two-parameter.

Research supported by Japan Society for the Promotion of Science. 
(A.4) There exists a constant $m>1$ such that for $u \in \mathbb{R}$

$$
f(u) u \geq m F(u):=m \int_{0}^{u} f(s) d s .
$$

The typical examples of $f(u)$ are as follows:

$$
\begin{aligned}
& f(u)=|u|^{p-1} u \quad \text { for } p>1 \text {, } \\
& f(u)=|u|^{p-1} u+|u|^{q-1} u \quad \text { for } p, q>1 \text {. }
\end{aligned}
$$

The purpose of this paper is to study the layer structure of the solutions to (1.1) for $\lambda \gg 1$ by using variational method. More precisely, we show the existence of the solutions $u_{\lambda}$ which have $2 n$ multiple interior layers in $I$ for $\lambda \gg 1$. We also determine the location of multiple interior layers of $u_{\lambda}$ as $\lambda \rightarrow \infty$. Furthermore, we show the existence of solutions $u_{\lambda}$ with boundary layers.

The equation (1.1) is motivated by the perturbed Sine-Gordon equation

$$
u_{t t}=u_{x x}-\sin u+f(u) \text { for } 0<x<\pi,
$$

which was recently studied by Bobenko and Kuksin [1]. They studied small amplitude solutions of nonlinear Klein-Gordon equation which was regarded as a perturbation of (1.2). We note that the solutions $u_{\lambda}$ considered here are not small amplitude solutions.

For one-parameter singular perturbation problems, the possible layer structure of the solutions was brought out in O'Malley [3]. For nonlinear twoparameter problems, it is known that in some cases layers (spike and boundary) appear (cf. [4], [6]). However, the problems of interior transition layers for nonlinear two-parameter problems do not seem to have been studied. Recently, Shibata [5] considered the equation (1.1) by means of a constrained minimization method, and obtained the existence of solutions $u_{\lambda}$ which has exactly two interior layers in $I$ as $\lambda \rightarrow \infty$. The result obtained in [5] is regarded as the first step to clarify the rich layer structure of the equation (1.1).

We explain the variational framework. We consider the variational problem $(\mathbf{M})$ subject to the constraint depending on $\lambda$ :

(M) Minimize

$$
L_{\lambda}(u):=\frac{1}{2} \int_{I}\left|u^{\prime}(t)\right|^{2} d t+\lambda \int_{I}(1-\cos u(t)) d t
$$

under the constraint

$$
u \in M_{\alpha}:=\left\{u \in H_{0}^{1}(I): K(u):=\int_{I} F(u(t)) d t=2 T F(\alpha)\right\},
$$

where $\alpha>0$ is a fixed constant, $H_{0}^{1}(I)$ is the usual real Sobolev space. 
Then by the Lagrange multiplier theorem, we obtain solution trios $\left(\lambda, \mu(\lambda), u_{\lambda}\right) \in$ $\mathbb{R}_{+}^{2} \times M_{\alpha}$ of (1.1) (and consequently $u_{\lambda} \in C^{2}(\bar{I})$ by a standard regularity theorem) corresponding to the problem $(\mathbf{M})$.

In Shibata [5], the following result was proved.

Theorem 0 ([5, Theorem]). Assume (A.1)-(A.4). Let $0<\alpha<2 \pi$ satisfy $F(\alpha)<F(2 \pi) / 2$. Then:

(i) $u_{\lambda} \rightarrow 2 \pi$ locally uniformly on $\left(-T_{\alpha, 0}, T_{\alpha, 0}\right)$ as $\lambda \rightarrow \infty$, where $T_{\alpha, 0}:=$ $F(\alpha) T / F(2 \pi)$.

(ii) $u_{\lambda} \rightarrow 0$ locally uniformly on $I \backslash\left[-T_{\alpha, 0}, T_{\alpha, 0}\right]$ as $\lambda \rightarrow \infty$.

(iii) $\mu(\lambda) \rightarrow 0$ as $\lambda \rightarrow \infty$.

Theorem 0 implies that if $F(\alpha)<F(2 \pi) / 2$, then the location of the interior layers of $u_{\lambda}$ tends to $\pm T_{\alpha, 0}$ as $\lambda \rightarrow \infty$.

We first remove the restriction $F(\alpha)<F(2 \pi) / 2$ in Theorem 0 . To do this, we introduce the condition (A.5.n) for a given $n \in \mathbb{N}$ :

$$
H(n):=F(2(n+1) \pi)-2 n F(2 n \pi)+2 \sum_{k=0}^{n-1} F(2 k \pi)>0 .
$$

Note that "Assume (A.5.n)" implies that the assumption (A.5.n) holds only for a given $n$. The example of $f$ which satisfies (A.1)-(A.5.n) for a fixed $n \in \mathbb{N}$ is $f(u)=|u|^{p-1} u$ for $p>p_{n}$, where $p_{n}>1$ is a constant depending on a given $n$.

Theorem 1. Assume (A.1)-(A.4) and (A.5.1). Let $0<\alpha<2 \pi$ satisfy $F(\alpha) \geq F(2 \pi) / 2$. Then the assertions (i)-(iii) in Theorem 0 hold.

Secondly, we show the existence of the solutions $u_{\lambda}$ which have $2(n+1)$ multiple interior transition layers at $t= \pm T_{\alpha, n}, \pm\left(T-T_{\alpha, n}\right), \pm\left(T-3 T_{\alpha, n}\right), \ldots, \pm(T-$ $\left.(2 n-1) T_{\alpha, n}\right)$ as $\lambda \rightarrow \infty$, where

$$
T_{\alpha, n}:=(F(\alpha)-F(2 n \pi)) T / H(n) .
$$

For $D \subset \mathbb{R}$, let $-D:=\{-t: t \in D\} \subset \mathbb{R}$ and $|D|$ be the Lebesgue measure of $D$.

Theorem 2. Let $n \in \mathbb{R}$ be given. Assume (A.1)-(A.4) and (A.5.n). If $\alpha$ satisfies $2 n \pi<\alpha<2(n+1) \pi$ and

$$
F(2 n \pi)<F(\alpha)<\frac{1}{2(n+1)} F(2(n+1) \pi)+\frac{1}{(n+1)} \sum_{k=0}^{n} F(2 k \pi),
$$

then as $\lambda \rightarrow \infty$ :

(i) $\left\|u_{\lambda}\right\|_{\infty}<2(n+1) \pi$.

(ii) $u_{\lambda} \rightarrow 2(n+1) \pi$ locally uniformly on $\left(-T_{\alpha, n}, T_{\alpha, n}\right)$.

(iii) $u_{\lambda} \rightarrow 2 n \pi$ locally uniformly on $\pm\left(T_{\alpha, n}, T-(2 n-1) T_{\alpha, n}\right)$. 
(iv) $u_{\lambda} \rightarrow 2 k \pi$ locally uniformly on $\pm\left(T-(2 k+1) T_{\alpha, n}, T-(2 k-1) T_{\alpha, n}\right)$ for $k=1, \ldots, n-1$.

(v) $u_{\lambda} \rightarrow 0$ locally uniformly on $\pm\left(T-T_{\alpha, n}, T\right]$.

(vi) There exist constants $C_{1}, C_{2}>0$ such that

$$
\mu(\lambda) \leq C_{1} \lambda e^{-C_{2} \sqrt{\lambda}} .
$$

Note that if (A.5.n) is satisfied, then there exists $\alpha>0$ which satisfies $2 n \pi<\alpha<2(n+1) \pi$ and (1.5) for $n$.

The rough idea of the proof of Theorems 2 is as follows. By using the variational characterization of $u_{\lambda}$, we find that the shape of $u_{\lambda}$ for $\lambda \gg 1$ is like step function, each height of the steps are $2 \pi$. We first establish an estimate $\left\|u_{\lambda}\right\|_{\infty}<2(n+1) \pi$ for $\lambda \gg 1$ by using (A.5.n). Then $u_{\lambda}$ must cross the line $u=2 \pi, \ldots, 2 n \pi$. By using this fact, we secondly establish that $\left|I_{\lambda, k}\right| \sim 2\left|I_{\lambda, 0}\right|$ for $\lambda \gg 1$, where $I_{\lambda, k} \subset(0, T)(k=1, \ldots, n-1)$ are the intervals on which $u_{\lambda} \rightarrow 2 k \pi$ locally uniformly as $\lambda \rightarrow \infty$. Finally, by using an estimate $\left\|u_{\lambda}\right\|_{\infty}<2(n+1) \pi$, we prove that $\left|I_{\lambda, 2(n+1)}\right| \sim\left|I_{\lambda, 0}\right|$ for $\lambda \gg 1$.

We next consider the case where the condition (1.5) does not hold. Namely, we consider $\alpha>0$ which satisfies $2 n \pi<\alpha<2(n+1) \pi$ and

$$
\frac{1}{2(n+1)} F(2(n+1) \pi)+\frac{1}{(n+1)} \sum_{k=0}^{n} F(2 k \pi) \leq F(\alpha) .
$$

In this case, $u_{\lambda}$ has multiple interior layers at $t= \pm\left(T-(2 k-1) S_{\alpha, n}\right)$ for $k=1, \ldots, n+1$, as $\lambda \rightarrow \infty$, where

$$
S_{\alpha, n}:=\frac{(F(2(n+1) \pi)-F(\alpha)) T}{(2 n+1) F(2(n+1) \pi)-2 \sum_{k=0}^{n} F(2 k \pi)} .
$$

Theorem 3. Let $n \in \mathbb{R}$ be given. Assume (A.1)-(A.4), (A.5.n) and (A.5. $n+$ 1). Let $2 n \pi<\alpha<2(n+1) \pi$ satisfy (1.7). Then as $\lambda \rightarrow \infty$ :

(i) $\left\|u_{\lambda}\right\|_{\infty} \rightarrow 2(n+1) \pi$.

(ii) $u_{\lambda} \rightarrow 2(n+1) \pi$ locally uniformly on $\left(-\left(T-(2 n+1) S_{\alpha, n}\right), T-(2 n+\right.$ 1) $\left.S_{\alpha, n}\right)$.

(iii) $u_{\lambda} \rightarrow 2 k \pi$ locally uniformly on $\pm\left(T-(2 k+1) S_{\alpha, n}, T-(2 k-1) S_{\alpha, n}\right)$ for $k=1, \ldots, n$.

(iv) $u_{\lambda} \rightarrow 0$ locally uniformly on $\pm\left(T-S_{\alpha, n}, T\right]$.

(v) The formula (1.6) holds.

To prove Theorem 3, we show that $\left|I_{\lambda, k}\right| \sim 2\left|I_{\lambda, 0}\right|$ for $k=1, \ldots, n$ and $\lambda \gg 1$. We also see from Theorems 2 and 3 that when $2 n \pi<\alpha<2(n+1) \pi$, there are two types of interior transition layers according to the range of $\alpha$.

Finally, we show the existence of solutions which have boundary layers. 
Theorem 4. Let $n \in \mathbb{N}$ be given. Assume (A.1)-(A.4) and (A.5.n). If $\alpha=2 n \pi$, then $\left\|u_{\lambda}\right\|_{\infty}<2(n+1) \pi$ for $\lambda \gg 1$ and $u_{\lambda} \rightarrow 2 n \pi$ locally uniformly on $(-T, 0) \cup(0, T)$ as $\lambda \rightarrow \infty$.

The remainder of this paper is organized as follows. In Section 2, we introduce the useful Lemmas which were obtained in Shibata [5] under the assumptions (A.1)-(A.4). In Section 3, we prove Theorem 2. The proof of the case $n=1$ is the main part of this section. In Section 4 through 6, we prove Theorems 1, 3 and 4, respectively. In Section 7, we prove some Lemmas introduced in Section 2 for completeness.

\section{Preliminaries}

In this section, we assume (A.1)-(A.4). For simplicity, we denote by $C$ the various positive constants independent of $\lambda$. A subsequence of a sequence is often denoted by the same notation as that of original sequence. We know by [2] that a solution $u$ of (1.1) satisfies the following properties:

$$
\begin{array}{rlrl}
u(t) & =u(-t) & & \text { for } t \in[0, T], \\
u^{\prime}(t) & <0 & & \text { for } \in(0, T], \\
u^{\prime}(0) & =0, u(0)=\|u\|_{\infty} . &
\end{array}
$$

For $0 \leq r \leq\left\|u_{\lambda}\right\|_{\infty}$, let $t_{r, \lambda} \in[0, T]$ satisfy $u_{\lambda}\left(t_{r, \lambda}\right)=r$, which exists uniquely by (2.2). The following notation will be used repeatedly. For a fixed $0<\varepsilon \ll 1$, let

$$
l_{\lambda, \varepsilon}:=t_{2 \pi, \lambda}-t_{2 \pi+\varepsilon, \lambda}, \quad m_{\lambda, \varepsilon}:=t_{2 \pi-\varepsilon, \lambda}-t_{2 \pi, \lambda}, \quad \delta_{\lambda, \epsilon}:=T-t_{\varepsilon, \lambda} .
$$

In what follows, we always fix $0<\varepsilon \ll 1$ first. Then let $\lambda \rightarrow \infty$. Therefore, the standard notation $o(1)$ will be used for $\lambda \gg 1$. Furthermore, the notation $l_{\lambda, \varepsilon}=\delta_{\lambda, \varepsilon}+O(\varepsilon)+o(1)$ (for instance) means that $\left|l_{\lambda, \varepsilon}-\delta_{\lambda, \varepsilon}\right| \leq C \varepsilon+o(1)$ for $0<\varepsilon \ll 1$ fixed and $\lambda \gg 1$.

Lemma 2.1. Assume that $(\lambda, \mu, u) \in \mathbb{R}_{+} \times \mathbb{R} \times C^{2}(\bar{I})$ satisfies (1.1). Then $\mu>0$. Further, for $t \in \bar{I}$,

(2.4) $\frac{1}{2} u^{\prime}(t)^{2}+\mu F(u(t))+\lambda \cos u(t)=\frac{1}{2} u^{\prime}(T)^{2}+\lambda=\mu F\left(\|u\|_{\infty}\right)+\lambda \cos \|u\|_{\infty}$.

Proof. Multiply the equation in (1.1) by $u^{\prime}(t)$. Then we have

$$
\left\{u^{\prime \prime}(t)+\mu f(u(t))-\lambda \sin u(t)\right\} u^{\prime}(t)=0, \quad t \in \bar{I} .
$$

This implies

$$
\frac{d}{d t}\left\{\frac{1}{2} u^{\prime}(t)^{2}+\mu F(u(t))+\lambda \cos u(t)\right\}=0, \quad t \in \bar{I} .
$$


Hence, for $t \in \bar{I}$,

$$
\frac{1}{2} u^{\prime}(t)^{2}+\mu F(u(t))+\lambda \cos u(t) \equiv \text { constant. }
$$

By putting $t=0, T$ in (2.5), we obtain (2.4) by (2.3). Then by (2.4), we obtain

$$
\mu F\left(\|u\|_{\infty}\right)=\frac{1}{2} u^{\prime}(T)^{2}+\lambda\left(1-\cos \|u\|_{\infty}\right)>0 .
$$

Since $F\left(\|u\|_{\infty}\right)>0$ by (A.1), $\mu>0$ follows from (2.6).

Lemma 2.2. Let $\alpha>0$ and $\lambda>0$ be fixed. Then there exists $\left(\mu(\lambda), u_{\lambda}\right) \in$ $\mathbb{R}_{+} \times\left(M_{\alpha} \cap C^{2}(\bar{I})\right)$ which satisfies (1.1) and $L_{\lambda}\left(u_{\lambda}\right)=\beta(\lambda):=\inf _{u \in M_{\alpha}} L_{\lambda}(u)$.

Lemma 2.3. Let $\alpha>0$ be fixed. Then $L_{\lambda}\left(u_{\lambda}\right) \leq C \lambda^{(m+2) / 2(m+1)}$ for $\lambda \gg 1$.

Lemma 2.4. Let $\alpha>0$ be fixed. Then $\mu(\lambda)=o(\lambda)$ for $\lambda \gg 1$.

Since Lemma 2.2 can be proved easily by choosing a minimizing sequence, we omit the proof. For the proof of Lemmas 2.3-2.4, see appendix (Section 7).

By Lemma 2.3, we obtain the following (2.7), which will be used later. Put $J_{\lambda, k, \delta}:=\left\{t \in I: 2(k-1) \pi+\delta<u_{\lambda}(t)<2 k \pi-\delta\right\}$ for $0<\delta \ll 1$ and $k \in \mathbb{N}$. By Lemma 2.3, as $\lambda \rightarrow \infty$,

$$
\begin{aligned}
\left|J_{\lambda, k, \delta}\right| & \leq \frac{1}{1-\cos \delta} \int_{J_{\lambda, k, \delta}}\left(1-\cos u_{\lambda}(t)\right) d t \\
& \leq \frac{\lambda^{-1}}{1-\cos \delta} L_{\lambda}\left(u_{\lambda}\right) \leq C \lambda^{-m /(2(m+1))} \rightarrow 0 .
\end{aligned}
$$

Lemma 2.5. Let $\alpha>0$ be fixed. Then $\left|u_{\lambda}^{\prime}(T)\right|^{2} / \lambda \rightarrow 0$ as $\lambda \rightarrow \infty$.

Proof. Integrate (2.4) over $I$. Then

$$
\frac{1}{2}\left\|u_{\lambda}^{\prime}\right\|_{2}^{2}+2 T \mu(\lambda) F(\alpha)=T u_{\lambda}^{\prime}(T)^{2}+\lambda \int_{I}\left(1-\cos u_{\lambda}(t)\right) d t .
$$

This along with Lemmas 2.3 and 2.4 implies that for $\lambda \gg 1$

$$
T u_{\lambda}^{\prime}(T)^{2} \leq \frac{1}{2}\left\|u_{\lambda}^{\prime}\right\|_{2}^{2}+2 T \mu(\lambda) F(\alpha)=o(\lambda) .
$$

Thus the proof is complete.

Lemma 2.6. Let $\alpha>0$ and $0<\varepsilon \ll 1$ be fixed. Then, for $\lambda \gg 1$,

$$
u_{\lambda}^{\prime}(T)^{2} \leq C \lambda e^{-2 \delta_{\lambda, \varepsilon} \sqrt{(1-2 \varepsilon) \lambda}} .
$$

ProOF. Since $u_{\lambda} \in M_{\alpha}$, we see that $u_{\lambda}(0)=\left\|u_{\lambda}\right\|_{\infty} \geq \alpha$. Therefore, there exists a unique $t_{\varepsilon, \lambda} \in[0, T]$ for $0<\varepsilon \ll 1$. Since $\sin \theta \geq(1-\varepsilon) \theta$ for $0 \leq \theta \leq \varepsilon$, by (1.1), we obtain

$$
u_{\lambda}^{\prime \prime}(t)+\mu(\lambda) f\left(u_{\lambda}(t)\right)=\lambda \sin u_{\lambda}(t) \geq(1-\varepsilon) \lambda u_{\lambda}(t) \quad \text { for } t \in\left[t_{\varepsilon, \lambda}, T\right] .
$$


Since $u_{\lambda}^{\prime}(t) \leq 0$ in $[0, T]$ by $(2.2)$, it follows from (2.10) that

$$
\left\{u_{\lambda}^{\prime \prime}(t)+\mu(\lambda) f\left(u_{\lambda}(t)\right)-(1-\varepsilon) \lambda u_{\lambda}(t)\right\} u_{\lambda}^{\prime}(t) \leq 0 \quad \text { for } t \in\left[t_{\varepsilon, \lambda}, T\right] .
$$

That is,

$$
\begin{aligned}
\frac{d S_{\lambda, 1}(t)}{d t}:=\frac{d}{d t}\left\{\frac{1}{2} u_{\lambda}^{\prime}(t)^{2}+\mu(\lambda) F\left(u_{\lambda}(t)\right)-\frac{(1-\varepsilon) \lambda u_{\lambda}(t)^{2}}{2}\right\} \leq 0 \\
\text { for } t \in\left[t_{\varepsilon, \lambda}, T\right] .
\end{aligned}
$$

This implies that $S_{\lambda, 1}(t)$ is non-increasing on $\left[t_{\varepsilon, \lambda}, T\right]$. Hence,

(2.12) $\frac{1}{2} u_{\lambda}^{\prime}(t)^{2}+\mu(\lambda) F\left(u_{\lambda}(t)\right)-\frac{(1-\varepsilon) \lambda u_{\lambda}(t)^{2}}{2} \geq \frac{1}{2} u_{\lambda}^{\prime}(T)^{2} \quad$ for $t \in\left[t_{\varepsilon, \lambda}, T\right]$.

By (A.3) and Lemma 2.4, we have

$$
\varepsilon \lambda u_{\lambda}(t)^{2} \geq 2 \mu(\lambda) F\left(u_{\lambda}(t)\right)
$$

for $t \in\left[t_{\varepsilon, \lambda}, T\right]$ and $\lambda \gg 1$. Then, by (2.12) and (2.13), we obtain

$$
-u_{\lambda}^{\prime}(t) \geq \sqrt{u_{\lambda}^{\prime}(T)^{2}+(1-2 \varepsilon) \lambda u_{\lambda}(t)^{2}} \quad \text { for } t \in\left[t_{\varepsilon, \lambda}, T\right] .
$$

Therefore, by (2.14),

$$
\begin{aligned}
\delta_{\lambda, \varepsilon} & =T-t_{\varepsilon, \lambda}=\int_{t_{\varepsilon, \lambda}}^{T} 1 d t \\
& \leq \int_{t_{\varepsilon, \lambda}}^{T} \frac{-u_{\lambda}^{\prime}(t)}{\sqrt{u_{\lambda}^{\prime}(T)^{2}+(1-2 \varepsilon) \lambda u_{\lambda}(t)^{2}}} d t \\
& =\int_{0}^{\varepsilon} \frac{d s}{\sqrt{u_{\lambda}^{\prime}(T)^{2}+(1-2 \varepsilon) \lambda s^{2}}} \\
& =\frac{1}{\sqrt{(1-2 \varepsilon) \lambda}} \log \left(\frac{\left|\varepsilon+\sqrt{\varepsilon^{2}+X_{\lambda, 1}^{2}}\right|}{X_{\lambda, 1}}\right)
\end{aligned}
$$

where $X_{\lambda, 1}:=\left|u_{\lambda}^{\prime}(T)\right| / \sqrt{(1-2 \varepsilon) \lambda}$. Since $X_{\lambda, 1} \rightarrow 0$ as $\lambda \rightarrow \infty$ by Lemma 2.5, (2.15) implies that $X_{\lambda, 1} e^{\delta_{\lambda, \varepsilon} \sqrt{(1-2 \varepsilon) \lambda}} \leq 3 \varepsilon$ for $\lambda \gg 1$. By this, we obtain (2.9). $\square$

LEMma 2.7. Let $\alpha>0$ and $0<\varepsilon \ll 1$ be fixed. Assume that there exists a subsequence $\left\{\lambda_{j}\right\}$ of $\{\lambda\}\left(\lambda_{j} \rightarrow \infty\right.$ as $\left.j \rightarrow \infty\right)$ such that $\left\|u_{\lambda_{j}}\right\|_{\infty} \geq 2 \pi$. Then

$$
m_{\lambda_{j}, \varepsilon}=t_{2 \pi-\varepsilon, \lambda_{j}}-t_{2 \pi, \lambda_{j}} \geq \sqrt{1-2 \varepsilon} \delta_{\lambda_{j}, \varepsilon}-o(1) \quad \text { for } \lambda_{j} \gg 1 .
$$

ProOF. Since $\left\|u_{\lambda_{j}}\right\|_{\infty} \geq 2 \pi$, we see that $t_{2 \pi, \lambda_{j}} \in[0, T]$ exists. Let $J_{j, \varepsilon}:=$ $\left[t_{2 \pi, \lambda_{j}}, t_{2 \pi-\varepsilon, \lambda_{j}}\right]$. Since $1-\cos \theta \leq \theta^{2} / 2$ for $\theta \geq 0$ and $\cos \left(2 \pi-u_{\lambda_{j}}(t)\right)=$ $\cos u_{\lambda_{j}}(t)$, we obtain by (2.4) that for $t \in J_{j, \varepsilon}$,

$$
\begin{aligned}
\frac{1}{2} u_{\lambda_{j}}^{\prime}(t)^{2} & =\frac{1}{2} u_{\lambda_{j}}^{\prime}(T)^{2}+\lambda_{j}\left(1-\cos u_{\lambda_{j}}(t)\right)-\mu\left(\lambda_{j}\right) F\left(u_{\lambda_{j}}(t)\right) \\
& \leq \frac{1}{2} u_{\lambda_{j}}^{\prime}(T)^{2}+\frac{1}{2} \lambda_{j}\left(2 \pi-u_{\lambda_{j}}(t)\right)^{2} .
\end{aligned}
$$


This implies

$$
-u_{\lambda_{j}}^{\prime}(t) \leq \sqrt{u_{\lambda_{j}}^{\prime}(T)^{2}+\lambda_{j}\left(2 \pi-u_{\lambda_{j}}(t)\right)^{2}}
$$

for $t \in J_{j, \varepsilon}$. By this and (2.9), we obtain

$$
\begin{aligned}
m_{\lambda_{j}, \varepsilon} & =t_{2 \pi-\varepsilon, \lambda_{j}}-t_{2 \pi, \lambda_{j}} \\
& \geq \int_{J_{j, \varepsilon}} \frac{-u_{\lambda_{j}}^{\prime}(t)}{\sqrt{u_{\lambda_{j}}^{\prime}(T)^{2}+\lambda_{j}\left(2 \pi-u_{\lambda_{j}}(t)\right)^{2}}} d t \\
& =\int_{0}^{\varepsilon} \frac{1}{\sqrt{u_{\lambda_{j}}^{\prime}(T)^{2}+\lambda_{j} s^{2}}} d s=\frac{1}{\sqrt{\lambda_{j}}} \log \left(\frac{\varepsilon+\sqrt{u_{\lambda_{j}}^{\prime}(T)^{2} / \lambda_{j}+\varepsilon^{2}}}{\left|u_{\lambda_{j}}^{\prime}(T)\right| / \sqrt{\lambda_{j}}}\right) \\
& \geq \frac{1}{\sqrt{\lambda_{j}}} \log \left(\frac{2 \varepsilon}{\left|u_{\lambda_{j}}^{\prime}(T)\right| / \sqrt{\lambda_{j}}}\right) \geq \sqrt{(1-2 \varepsilon)} \delta_{\lambda_{j}, \varepsilon}-o(1) .
\end{aligned}
$$

Thus the proof is complete.

\section{Proof of Theorem 2}

The first aim of this section is to prove Theorem 2(i) for $n=1$ in Lemma 3.8. To do this, we compare $\left|t_{4 \pi-\varepsilon, \lambda}-t_{4 \pi, \lambda}\right|$ with $l_{\lambda, \varepsilon}, m_{\lambda, \varepsilon}, \delta_{\lambda, \varepsilon}$ in Lemmas 3.3, 3.5 and 3.7.

Lemma 3.1. Assume (A.1)-(A.4). Let $\alpha>0$ and $0<\varepsilon \ll 1$ be fixed. Then, for $\lambda \gg 1$,

$$
u_{\lambda}^{\prime}(T)^{2} \geq C_{\varepsilon} \lambda e^{-2 \delta_{\lambda, \varepsilon} \sqrt{\lambda}}
$$

Proof. By (1.1), we obtain

$$
u_{\lambda}^{\prime \prime}(t)+\mu(\lambda) f\left(u_{\lambda}(t)\right)=\lambda \sin u_{\lambda}(t) \leq \lambda u_{\lambda}(t) \quad \text { for } t \in\left[t_{\lambda, \varepsilon}, T\right] .
$$

By this and (2.2), we obtain

$$
\left\{u_{\lambda}^{\prime \prime}(t)+\mu(\lambda) f\left(u_{\lambda}(t)\right)-\lambda u_{\lambda}(t)\right\} u_{\lambda}^{\prime}(t) \geq 0 \quad \text { for } t \in\left[t_{\lambda, \varepsilon}, T\right] .
$$

That is,

$$
\frac{d S_{\lambda, 2}(t)}{d t}:=\frac{d}{d t}\left\{\frac{1}{2} u_{\lambda}^{\prime}(t)^{2}+\mu(\lambda) F\left(u_{\lambda}(t)\right)-\frac{\lambda u_{\lambda}(t)^{2}}{2}\right\} \geq 0 \quad \text { for } t \in\left[t_{\lambda, \varepsilon}, T\right] .
$$

This implies that $S_{\lambda, 2}(t)$ is increasing on $\left[t_{\lambda, \varepsilon}, T\right]$. Hence,

$$
\frac{1}{2} u_{\lambda}^{\prime}(t)^{2}+\mu(\lambda) F\left(u_{\lambda}(t)\right)-\frac{\lambda u_{\lambda}(t)^{2}}{2} \leq \frac{1}{2} u_{\lambda}^{\prime}(T)^{2} \quad \text { for } t \in\left[t_{\lambda, \varepsilon}, T\right] .
$$

Then, for $t \in\left[t_{\lambda, \varepsilon}, T\right]$,

$$
-u_{\lambda}^{\prime}(t) \leq \sqrt{u_{\lambda}^{\prime}(T)^{2}+\lambda u_{\lambda}(t)^{2}-2 \mu(\lambda) F\left(u_{\lambda}(t)\right)} \leq \sqrt{u_{\lambda}^{\prime}(T)^{2}+\lambda u_{\lambda}(t)^{2}}
$$


Therefore, by (3.2),

$$
\begin{aligned}
\delta_{\lambda, \varepsilon} & =T-t_{\varepsilon, \lambda}=\int_{t_{\varepsilon, \lambda}}^{T} 1 d t \geq \int_{t_{\varepsilon, \lambda}}^{T} \frac{-u_{\lambda}^{\prime}(t)}{\sqrt{u_{\lambda}^{\prime}(T)^{2}+\lambda u_{\lambda}(t)^{2}}} d t \\
& =\int_{0}^{\varepsilon} \frac{d s}{\sqrt{u_{\lambda}^{\prime}(T)^{2}+\lambda s^{2}}}=\frac{1}{\sqrt{\lambda}} \log \left(\frac{\left|\varepsilon+\sqrt{\varepsilon^{2}+X_{\lambda, 2}^{2}}\right|}{X_{\lambda, 2}}\right) \geq \frac{1}{\sqrt{\lambda}} \log \left(\frac{2 \varepsilon}{X_{\lambda, 2}}\right),
\end{aligned}
$$

where $X_{\lambda, 2}:=\left|u_{\lambda}^{\prime}(T)\right| / \sqrt{\lambda}$. This implies (3.1).

Lemma 3.2. Assume (A.1)-(A.4). Let $\alpha>0$ and $0<\varepsilon \ll 1$ be fixed. Suppose that there exists a subsequence $\left\{\lambda_{j}\right\}_{j=1}^{\infty}$ such that $\lambda_{j} \rightarrow \infty$ as $j \rightarrow \infty$ and $\left\|u_{\lambda_{j}}\right\|_{\infty} \geq 4 \pi$. Then

$$
u_{\lambda_{j}}^{\prime}\left(t_{2 \pi, \lambda_{j}}\right)^{2} \leq C \lambda_{j} e^{-2 l_{\lambda_{j}, \varepsilon} \sqrt{(1-\varepsilon) \lambda_{j}}} .
$$

Proof. For convenience, we write $\lambda=\lambda_{j}$. Put $t=T, t_{2 \pi, \lambda}, t_{4 \pi, \lambda}$ in (2.4). Then we obtain

$$
\begin{aligned}
\frac{1}{2} u_{\lambda}^{\prime}(t)^{2}+\mu(\lambda) F\left(u_{\lambda}(t)\right) & +\lambda \cos u_{\lambda}(t)=\frac{1}{2} u_{\lambda}^{\prime}\left(t_{4 \pi, \lambda}\right)^{2}+\mu(\lambda) F(4 \pi)+\lambda \\
= & \frac{1}{2} u_{\lambda}^{\prime}\left(t_{2 \pi, \lambda}\right)^{2}+\mu(\lambda) F(2 \pi)+\lambda=\frac{1}{2} u_{\lambda}^{\prime}(T)^{2}+\lambda
\end{aligned}
$$

This implies

$$
\mu(\lambda)(F(4 \pi)-F(2 \pi)) \leq \frac{1}{2} u_{\lambda}^{\prime}\left(t_{2 \pi, \lambda}\right)^{2} .
$$

In particular, by this and Lemma 2.4 , for $\lambda \gg 1$, we obtain

$$
\frac{\mu(\lambda)^{2}}{\lambda}=o(1) \mu(\lambda) \ll u_{\lambda}^{\prime}\left(t_{2 \pi, \lambda}\right)^{2} .
$$

For $t \in\left[t_{2 \pi+\varepsilon, \lambda}, t_{2 \pi, \lambda}\right]$, we have, by (1.1),

$$
u_{\lambda}^{\prime \prime}(t)+\mu(\lambda) f\left(u_{\lambda}(t)\right)=\lambda \sin u_{\lambda}(t)=\lambda \sin \left(u_{\lambda}(t)-2 \pi\right) \geq \lambda(1-\varepsilon)\left(u_{\lambda}(t)-2 \pi\right) .
$$

Therefore, by (2.2), for $t \in\left[t_{2 \pi+\varepsilon, \lambda}, t_{2 \pi, \lambda}\right]$, we have

$$
\left\{u_{\lambda}^{\prime \prime}(t)+\mu(\lambda) f\left(u_{\lambda}(t)\right)-\lambda(1-\varepsilon)\left(u_{\lambda}(t)-2 \pi\right)\right\} u_{\lambda}^{\prime}(t) \leq 0 .
$$

That is,

$$
\frac{d S_{\lambda, 3}(t)}{d t}:=\frac{d}{d t}\left\{\frac{1}{2} u_{\lambda}^{\prime}(t)^{2}+\mu(\lambda) F\left(u_{\lambda}(t)\right)-\frac{1-\varepsilon}{2} \lambda\left(u_{\lambda}-2 \pi\right)^{2}\right\} \leq 0 .
$$

Hence, $S_{\lambda, 3}(t)$ is decreasing in $\left[t_{2 \pi+\varepsilon, \lambda}, t_{2 \pi, \lambda}\right]$. Then we obtain

$$
\frac{1}{2} u_{\lambda}^{\prime}(t)^{2}+\mu(\lambda) F\left(u_{\lambda}(t)\right)-\frac{1-\varepsilon}{2} \lambda\left(u_{\lambda}-2 \pi\right)^{2} \geq \frac{1}{2} u_{\lambda}^{\prime}\left(t_{2 \pi, \lambda}\right)^{2}+\mu(\lambda) F(2 \pi)
$$

for $\left[t_{2 \pi+\varepsilon, \lambda}, t_{2 \pi, \lambda}\right]$. Then by this, (3.6) and the inequality

$$
F(u)-F(2 \pi) \leq C(1-\varepsilon)(u-2 \pi) \text { for } 2 \pi \leq u \leq 2 \pi+\varepsilon,
$$


for $\left[t_{2 \pi+\varepsilon, \lambda}, t_{2 \pi, \lambda}\right]$, we obtain

$$
\begin{aligned}
\frac{1}{2} u_{\lambda}^{\prime}(t)^{2} & \geq \frac{1}{2} u_{\lambda}^{\prime}\left(t_{2 \pi, \lambda}\right)^{2}+\frac{1-\varepsilon}{2} \lambda\left(u_{\lambda}(t)-2 \pi\right)^{2}-\mu(\lambda)\left(F\left(u_{\lambda}(t)\right)-F(2 \pi)\right) \\
& \geq \frac{1}{2} u_{\lambda}^{\prime}\left(t_{2 \pi, \lambda}\right)^{2}+\frac{1-\varepsilon}{2} \lambda\left\{\left(u_{\lambda}(t)-2 \pi\right)^{2}-2 C \frac{\mu(\lambda)}{\lambda}\left(u_{\lambda}(t)-2 \pi\right)\right\} \\
& =\frac{1}{2} u_{\lambda}^{\prime}\left(t_{2 \pi, \lambda}\right)^{2}+\frac{1-\varepsilon}{2} \lambda\left\{\left(u_{\lambda}(t)-2 \pi\right)-\frac{C \mu(\lambda)}{\lambda}\right\}^{2}-\frac{1-\varepsilon}{2} C^{2} \frac{\mu(\lambda)^{2}}{\lambda} \\
& \geq C u_{\lambda}^{\prime}\left(t_{2 \pi, \lambda}\right)^{2}+\frac{1-\varepsilon}{2} \lambda\left\{\left(u_{\lambda}(t)-2 \pi\right)-\frac{C \mu(\lambda)}{\lambda}\right\}^{2} .
\end{aligned}
$$

This implies

$$
\text { (3.8) } \begin{aligned}
l_{\lambda, \varepsilon} & =\int_{t_{2 \pi+\varepsilon, \lambda}}^{t_{2 \pi, \lambda}} 1 d t \\
& \leq \int_{t_{2 \pi+\varepsilon, \lambda}}^{t_{2 \pi, \lambda}} \frac{-u_{\lambda}^{\prime}(t)}{\sqrt{2 C u_{\lambda}^{\prime}\left(t_{2 \pi, \lambda}\right)^{2}+(1-\varepsilon) \lambda\left\{\left(u_{\lambda}(t)-2 \pi\right)-C \mu(\lambda) / \lambda\right\}^{2}}} d t \\
& =\int_{-C \mu(\lambda) / \lambda}^{\varepsilon-C \mu(\lambda) / \lambda} \frac{1}{\sqrt{2 C u_{\lambda}^{\prime}\left(t_{2 \pi, \lambda}\right)^{2}+(1-\varepsilon) \lambda s^{2}}} d s \\
& =K_{\lambda}:=\frac{1}{\sqrt{(1-\varepsilon) \lambda}} \int_{-C \mu(\lambda) / \lambda}^{\varepsilon-C \mu(\lambda) / \lambda} \frac{1}{\sqrt{s^{2}+X_{\lambda, 3}^{2}}} d s,
\end{aligned}
$$

where $X_{\lambda, 3}=\sqrt{2 C}\left|u_{\lambda}^{\prime}\left(t_{2 \pi, \lambda}\right)\right| / \sqrt{(1-\varepsilon) \lambda}$. By (3.4), we have

$$
\frac{1}{2} u_{\lambda}^{\prime}\left(t_{2 \pi, \lambda}\right)^{2} \leq \frac{1}{2} u_{\lambda}^{\prime}\left(t_{2 \pi, \lambda}\right)^{2}+\mu(\lambda) F(2 \pi)=\frac{1}{2} u_{\lambda}^{\prime}(T)^{2} .
$$

By this and Lemma 2.5, we see that $X_{\lambda, 3}^{2} \rightarrow 0$ as $\lambda \rightarrow \infty$. By (3.6), for $\lambda \gg 1$, we have

By this and (3.8), we obtain

$$
-\frac{C \mu(\lambda)}{\lambda}+\sqrt{\frac{C^{2} \mu(\lambda)^{2}}{\lambda^{2}}+X_{\lambda, 3}^{2}} \geq \frac{X_{\lambda, 3}}{2} .
$$

$$
\begin{aligned}
l_{\lambda, \varepsilon} & \leq K_{\lambda}=\frac{1}{\sqrt{(1-\varepsilon) \lambda}}\left[\log \left|s+\sqrt{s^{2}+X_{\lambda, 4}^{2}}\right|\right]_{-C \mu(\lambda) / \lambda}^{\varepsilon-C \mu(\lambda) / \lambda} \\
& \leq \frac{1}{\sqrt{(1-\varepsilon) \lambda}}\left\{\log 3 \varepsilon-\log \left|-\frac{C \mu(\lambda)}{\lambda}+\sqrt{\frac{C^{2} \mu(\lambda)^{2}}{\lambda^{2}}+X_{\lambda, 3}^{2}}\right|\right\} \\
& \leq \frac{1}{\sqrt{(1-\varepsilon) \lambda}}\left\{\log 3 \varepsilon-\log \left|X_{\lambda, 3}\right| / 2\right\} \\
& \leq \frac{1}{\sqrt{(1-\varepsilon) \lambda}} \log \frac{\sqrt{(1-\varepsilon) \lambda}}{\left|\sqrt{C / 2} u_{\lambda}^{\prime}\left(t_{2 \pi, \lambda}\right)\right|} .
\end{aligned}
$$

By this, we obtain (3.3). 
Lemma 3.3. Assume (A.1)-(A.4). Let $\alpha>0$ and $0<\varepsilon \ll 1$ be fixed. Suppose that there exists a subsequence $\left\{\lambda_{j}\right\}_{j=1}^{\infty}$ such that $\lambda_{j} \rightarrow \infty$ as $j \rightarrow \infty$ and $\left\|u_{\lambda_{j}}\right\|_{\infty} \geq 4 \pi$. Then

$$
t_{4 \pi-\varepsilon, \lambda_{j}}-t_{4 \pi, \lambda_{j}} \geq \sqrt{(1-\varepsilon)} l_{\lambda_{j}, \varepsilon}-o(1) .
$$

Lemma 3.3 follows from Lemma 3.2 and the same calculation as those used in Lemma 2.7. Hence we omit the proof.

Lemma 3.4. Assume (A.1)-(A.4). Let $\alpha>0$ and $0<\varepsilon \ll 1$ be fixed. Suppose that there exists a subsequence $\left\{\lambda_{j}\right\}$ such that $\lambda_{j} \rightarrow \infty$ as $j \rightarrow \infty$, and $\left\|u_{\lambda_{j}}\right\|_{\infty} \geq 2 \pi$. Then

$$
u_{\lambda_{j}}^{\prime}\left(t_{2 \pi, \lambda}\right)^{2} \leq C \lambda_{j} e^{-2 m_{\lambda_{j}, \varepsilon} \sqrt{(1-\varepsilon) \lambda}} .
$$

Proof. We write $\lambda=\lambda_{j}$, for short. For $t \in\left[t_{2 \pi, \lambda}, t_{2 \pi-\varepsilon, \lambda}\right]$, by (1.1), we have

$$
\begin{aligned}
u_{\lambda}^{\prime \prime}(t)+\mu(\lambda) f\left(u_{\lambda}(t)\right) & =\lambda \sin u_{\lambda}(t)=-\lambda \sin \left(2 \pi-u_{\lambda}(t)\right) \\
& \leq-\lambda(1-\varepsilon)\left(2 \pi-u_{\lambda}(t)\right)=\lambda(1-\varepsilon)\left(u_{\lambda}(t)-2 \pi\right) .
\end{aligned}
$$

Then for $t \in\left[t_{2 \pi, \lambda}, t_{2 \pi-\varepsilon, \lambda}\right]$, by (2.2) and (3.13), we obtain

$$
\left\{u_{\lambda}^{\prime \prime}(t)+\mu(\lambda) f\left(u_{\lambda}(t)\right)-\lambda(1-\varepsilon)\left(u_{\lambda}(t)-2 \pi\right)\right\} u_{\lambda}^{\prime}(t) \geq 0 .
$$

This implies that for $t \in\left[t_{2 \pi, \lambda}, t_{2 \pi-\varepsilon, \lambda}\right]$,

$$
\frac{d S_{\lambda, 4}(t)}{d t}:=\frac{d}{d t}\left\{\frac{1}{2} u_{\lambda}^{\prime}(t)+\mu(\lambda) F\left(u_{\lambda}(t)\right)-\frac{1-\varepsilon}{2}\left(u_{\lambda}(t)-2 \pi\right)^{2}\right\} \geq 0 .
$$

So $S_{\lambda, 4}(t)$ is non-decreasing in $\left[t_{2 \pi, \lambda}, t_{2 \pi-\varepsilon, \lambda}\right]$. Therefore, for $t \in\left[t_{2 \pi, \lambda}, t_{2 \pi-\varepsilon, \lambda}\right]$, we obtain

$$
\frac{1}{2} u_{\lambda}^{\prime}(t)^{2}+\mu(\lambda) F\left(u_{\lambda}(t)\right)-\frac{1-\varepsilon}{2}\left(u_{\lambda}(t)-2 \pi\right)^{2} \geq \frac{1}{2} u_{\lambda}^{\prime}\left(t_{2 \pi, \lambda}\right)^{2}+\mu(\lambda) F(2 \pi) .
$$

This implies

$$
\frac{1}{2} u_{\lambda}^{\prime}(t)^{2} \geq \frac{1}{2} u_{\lambda}\left(t_{2 \pi, \lambda}\right)^{2}+\frac{1-\varepsilon}{2}\left(u_{\lambda}(t)-2 \pi\right)^{2} .
$$

By (3.14) and the same calculation as those used in the proof of Lemma 2.6, we obtain our conclusion.

Lemma 3.5. Assume (A.1)-(A.4). Let $\alpha>0$ and $0<\varepsilon \ll 1$ be fixed. Suppose that there exists a subsequence $\left\{\lambda_{j}\right\}$ such that $\lambda_{j} \rightarrow \infty$ as $j \rightarrow \infty$, and $\left\|u_{\lambda_{j}}\right\|_{\infty} \geq 4 \pi$. Then

$$
t_{4 \pi-\varepsilon, \lambda_{j}}-t_{4 \pi, \lambda_{j}} \geq \sqrt{1-\varepsilon} m_{\lambda_{j}, \varepsilon}-o(1) \quad \text { for } \lambda_{j} \gg 1 .
$$

Lemma 3.5 can be proved by using Lemma 3.4 and the same arguments as those in the proof of Lemma 2.7. Therefore, we omit the proof. 
Lemma 3.6. Assume (A.1)-(A.4). Let $\alpha>0$ and $0<\varepsilon \ll 1$ be fixed. Assume that there exists a subsequence $\left\{\lambda_{j}\right\}$ such that $\lambda_{j} \rightarrow \infty$ as $j \rightarrow \infty$, and $\left\|u_{\lambda_{j}}\right\|_{\infty} \geq 2 \pi+\varepsilon$. Then

$$
l_{\lambda_{j}, \varepsilon}=t_{2 \pi, \lambda_{j}}-t_{2 \pi+\varepsilon, \lambda_{j}} \geq \sqrt{1-2 \varepsilon} \delta_{\lambda_{j}, \varepsilon}-o(1) \quad \text { for } \lambda_{j} \gg 1
$$

Proof. We abreviate $\lambda_{j}$ as $\lambda$. For $t \in\left[t_{2 \pi+\varepsilon, \lambda}, t_{2 \pi, \lambda}\right]$, by (2.4), we obtain

$$
\begin{aligned}
\frac{1}{2} u_{\lambda}^{\prime}(t)^{2} & \leq \frac{1}{2} u_{\lambda}^{\prime}(T)^{2}+\lambda\left(1-\cos u_{\lambda}(t)\right)=\frac{1}{2} u_{\lambda}^{\prime}(T)^{2}+\lambda\left(1-\cos \left(u_{\lambda}(t)-2 \pi\right)\right) \\
& \leq \frac{1}{2} u_{\lambda}^{\prime}(T)^{2}+\frac{1}{2} \lambda\left(u_{\lambda}(t)-2 \pi\right)^{2} .
\end{aligned}
$$

This implies

$$
-u_{\lambda}^{\prime}(t) \leq \sqrt{\lambda\left(u_{\lambda}(t)-2 \pi\right)^{2}+u_{\lambda}^{\prime}(T)^{2}}
$$

for $t \in\left[t_{2 \pi+\varepsilon, \lambda}, t_{2 \pi, \lambda}\right]$. This yields

$$
\begin{aligned}
l_{\lambda, \varepsilon} & =t_{2 \pi, \lambda}-t_{2 \pi+\varepsilon, \lambda} \\
& \geq \int_{t_{2 \pi+\varepsilon, \lambda}}^{t_{2 \pi, \lambda}} \frac{-u_{\lambda}^{\prime}(t)}{\sqrt{\lambda\left(u_{\lambda}(t)-2 \pi\right)^{2}+u_{\lambda}^{\prime}(T)^{2}}} d t=\int_{0}^{\varepsilon} \frac{1}{\sqrt{\lambda s^{2}+u_{\lambda}^{\prime}(T)^{2}}} d t .
\end{aligned}
$$

By using this and the same calculation as that in the proof of Lemma 2.7, we obtain (3.16).

Lemma 3.7. Assume (A.1)-(A.4). Let $\alpha>0$ and $0<\varepsilon \ll 1$ be fixed. Assume that there exists a subsequence $\left\{\lambda_{j}\right\}$ such that $\lambda_{j} \rightarrow \infty$ as $j \rightarrow \infty$, and $\left\|u_{\lambda_{j}}\right\|_{\infty} \geq 4 \pi$. Then

$$
t_{4 \pi-\varepsilon, \lambda_{j}}-t_{4 \pi, \lambda_{j}} \geq \sqrt{1-2 \varepsilon} \delta_{\lambda_{j}, \varepsilon}-o(1) \quad \text { for } \lambda_{j} \gg 1
$$

Lemma 3.7 can be proved by exactly the same arguments as those used in the proof of Lemma 2.7. Hence we omit the proof.

Now we prove Theorem 2(i) for $n=1$ in the following Lemma 3.8.

Lemma 3.8. Assume (A.1)-(A.4) and (A.5.1). Let $2 \pi<\alpha<4 \pi$ which satisfies (1.5) for $n=1$ be fixed. Then $\left\|u_{\lambda}\right\|_{\infty}<4 \pi$ for $\lambda \gg 1$.

Proof. We assume that there exists a subsequence of $\{\lambda\}$, denoted by $\{\lambda\}$ again, such that $\lambda \rightarrow \infty$ and $\left\|u_{\lambda}\right\|_{\infty} \geq 4 \pi$, and derive a contradiction. Let $0<\varepsilon \ll 1$ be fixed. By (2.7), we see that as $\lambda \rightarrow \infty$

$$
\left|t_{\varepsilon, \lambda}-t_{2 \pi-\varepsilon, \lambda}\right|,\left|t_{2 \pi+\varepsilon, \lambda}-t_{4 \pi-\varepsilon, \lambda}\right| \rightarrow 0 .
$$


Then by (3.18), we obtain

$$
\begin{aligned}
T= & T-t_{\varepsilon, \lambda}+\left(t_{\varepsilon, \lambda}-t_{2 \pi-\varepsilon, \lambda}\right)+\left(t_{2 \pi-\varepsilon, \lambda}-t_{2 \pi, \lambda}\right) \\
& +\left(t_{2 \pi, \lambda}-t_{2 \pi+\varepsilon, \lambda}\right)+\left(t_{2 \pi+\varepsilon, \lambda}-t_{4 \pi-\varepsilon, \lambda}\right)+t_{4 \pi-\varepsilon, \lambda} \\
= & \delta_{\lambda, \varepsilon}+l_{\lambda, \varepsilon}+m_{\lambda, \varepsilon}+t_{4 \pi-\varepsilon, \lambda}+\left(t_{\varepsilon, \lambda}-t_{2 \pi-\varepsilon, \lambda}\right) \\
& +\left(t_{2 \pi+\varepsilon, \lambda}-t_{4 \pi-\varepsilon, \lambda}\right) \\
= & \delta_{\lambda, \varepsilon}+l_{\lambda, \varepsilon}+m_{\lambda, \varepsilon}+t_{4 \pi-\varepsilon, \lambda}+o(1) .
\end{aligned}
$$

Therefore, by (3.19), Lemmas 3.3, 3.5 and 3.7, we have

$$
T \leq 3\left(t_{4 \pi-\varepsilon, \lambda}-t_{4 \pi, \lambda}\right)+t_{4 \pi-\varepsilon, \lambda}+O(\varepsilon)+o(1) \leq 4 t_{4 \pi-\varepsilon, \lambda}+O(\varepsilon)+o(1) .
$$

This implies that for $\lambda \gg 1$

$$
T / 4 \leq t_{4 \pi-\varepsilon, \lambda}+O(\varepsilon)+o(1) .
$$

On the other hand, by Lemmas 2.7, 3.6, (3.19) and (3.20), we have

$$
\begin{aligned}
3 \delta_{\lambda, \varepsilon} & \leq \delta_{\lambda, \varepsilon}+m_{\lambda, \varepsilon}+l_{\lambda, \varepsilon}+O(\varepsilon)+o(1) \\
& =T-t_{4 \pi-\varepsilon, \lambda}+O(\varepsilon)+o(1) \leq 3 T / 4+O(\varepsilon)+o(1) .
\end{aligned}
$$

This implies that for $\lambda \gg 1$

$$
\delta_{\lambda, \varepsilon} \leq T / 4+O(\varepsilon)+o(1)
$$

We know

$$
\begin{aligned}
T F(\alpha)=\sum_{k=1}^{4} B_{k, \lambda, \varepsilon}:=\int_{0}^{T / 4-C \varepsilon} F\left(u_{\lambda}(t)\right) d t+\int_{T / 4-C \varepsilon}^{t_{2 \pi-\varepsilon, \lambda}} F\left(u_{\lambda}(t)\right) d t \\
+\int_{t_{2 \pi-\varepsilon, \lambda}}^{t_{\varepsilon, \lambda}} F\left(u_{\lambda}(t)\right) d t+\int_{t_{\varepsilon, \lambda}}^{T} F\left(u_{\lambda}(t)\right) d t .
\end{aligned}
$$

By (3.18), we obtain that $B_{3, \lambda, \varepsilon} \rightarrow 0$ as $\lambda \rightarrow \infty$. It is clear that $B_{4, \lambda, \varepsilon} \leq C \varepsilon$. By (3.20), we see that $T / 4-C \varepsilon \leq t_{4 \pi-\varepsilon, \lambda}$ for $\lambda \gg 1$. Then by this, we obtain

$$
B_{1, \lambda, \varepsilon} \geq F(4 \pi-\varepsilon)\left(\frac{T}{4}-C \varepsilon\right) \geq \frac{T F(4 \pi)}{4}-C \varepsilon .
$$

By (3.18) and (3.21), we obtain

$$
\begin{aligned}
B_{2, \lambda, \varepsilon} & \geq F(2 \pi-\varepsilon)\left(t_{2 \pi-\varepsilon, \lambda}-T / 4+C \varepsilon\right) \\
& =F(2 \pi-\varepsilon)\left(\left(t_{2 \pi-\varepsilon, \lambda}-t_{\varepsilon, \lambda}\right)+T-\delta_{\lambda, \varepsilon}-T / 4+C \varepsilon\right) \\
& \geq \frac{T F(2 \pi)}{2}-C \varepsilon-o(1) .
\end{aligned}
$$

By these inequalities and (3.22), we obtain

$$
F(\alpha) \geq \frac{F(4 \pi)}{4}+\frac{F(2 \pi)}{2}-C \varepsilon-o(1) .
$$


Choose $\varepsilon$ sufficiently small. Then this contradicts (1.5) for $n=1$. Thus the proof is complete.

In the following Lemmas $3.9-3.10$, we estimate $l_{\lambda, \varepsilon}$ and $m_{\lambda, \varepsilon}$ by $\delta_{\lambda, \varepsilon}$ from above. To do this, the following inequality (3.24) plays an important role:

$$
C \mu(\lambda) \leq u_{\lambda}^{\prime}\left(t_{2 \pi, \lambda}\right)^{2}
$$

Lemma 3.9. Assume (A.1)-(A.4). Let $2 \pi<\alpha<4 \pi$ and $0<\varepsilon \ll 1$ be fixed. Assume that $2 \pi<\left\|u_{\lambda}\right\|_{\infty}<4 \pi$ for $\lambda \gg 1$. Suppose that there exists a subsequence $\left\{\lambda_{j}\right\}_{j=1}^{\infty}$ such that $\lambda_{j} \rightarrow \infty$ as $j \rightarrow \infty$ and satisfies (3.24). Then for $j \gg 1$

$$
m_{\lambda_{j}, \varepsilon}=t_{2 \pi-\varepsilon, \lambda_{j}}-t_{2 \pi, \lambda_{j}} \leq \delta_{\lambda_{j}, \varepsilon}+O(\varepsilon)+o(1)
$$

ProOF. We write $\lambda=\lambda_{j}$ for short. By (2.4), for $t \in\left[t_{2 \pi, \lambda}, t_{2 \pi-\varepsilon, \lambda}\right]$, we have

$$
\begin{aligned}
\frac{1}{2} u_{\lambda}^{\prime}(t)^{2} & =\frac{1}{2} u_{\lambda}^{\prime}\left(t_{2 \pi}\right)^{2}+\lambda\left(1-\cos u_{\lambda}(t)\right)+\mu(\lambda)\left(F(2 \pi)-F\left(u_{\lambda}\right)\right) \\
& \geq \frac{1}{2} u_{\lambda}^{\prime}\left(t_{2 \pi}\right)^{2}+\frac{(1-C \varepsilon)}{2} \lambda\left(2 \pi-u_{\lambda}(t)\right)^{2}
\end{aligned}
$$

This implies

$$
\begin{aligned}
m_{\lambda, \varepsilon} & =\int_{t_{2 \pi, \lambda}}^{t_{2 \pi-\varepsilon, \lambda}} 1 d t \\
& \leq \int_{t_{2 \pi, \lambda}}^{t_{2 \pi-\varepsilon, \lambda}} \frac{-u_{\lambda}^{\prime}(t)}{\sqrt{u_{\lambda}^{\prime}\left(t_{2 \pi, \lambda}\right)^{2}+\lambda(1-C \varepsilon)\left(2 \pi-u_{\lambda}(t)\right)^{2}}} d t \\
& =\int_{0}^{\varepsilon} \frac{1}{\sqrt{\lambda(1-C \varepsilon) s^{2}+u_{\lambda}^{\prime}\left(t_{2 \pi, \varepsilon}\right)^{2}}} d s \\
& =K_{\lambda}:=\frac{1}{\sqrt{(1-C \varepsilon) \lambda}} \int_{0}^{\varepsilon} \frac{1}{\sqrt{s^{2}+X_{\lambda, 4}^{2}}} d s,
\end{aligned}
$$

where $X_{\lambda, 4}:=\left|u_{\lambda}^{\prime}\left(t_{2 \pi, \lambda}\right)\right| / \sqrt{(1-C \varepsilon) \lambda}$. Then by Lemma 2.5 and (3.9), we see that $X_{\lambda, 4}^{2} \rightarrow 0$ as $\lambda \rightarrow \infty$. Then by direct calculation, we have

$$
\begin{aligned}
K_{\lambda} & =\frac{1}{\sqrt{(1-C \varepsilon) \lambda}} \log \left|\frac{\varepsilon+\sqrt{\varepsilon^{2}+X_{\lambda, 4}^{2}}}{X_{\lambda, 4}}\right| \\
& \leq \frac{1}{\sqrt{(1-C \varepsilon) \lambda}}\left(C-\log \left|u_{\lambda}^{\prime}\left(t_{2 \pi, \lambda}\right)\right|+\log \sqrt{\lambda}\right) .
\end{aligned}
$$


By (2.4), (3.24), Lemma 3.1 and Lemma 3.8, we obtain

$$
\begin{aligned}
C u_{\lambda}^{\prime}\left(t_{2 \pi, \lambda}\right)^{2} & \geq \mu(\lambda) F(4 \pi) \geq \mu(\lambda) F\left(\left\|u_{\lambda}\right\|_{\infty}\right) \\
& =\frac{1}{2} u_{\lambda}^{\prime}(T)^{2}+\lambda\left(1-\cos \left\|u_{\lambda}\right\|_{\infty}\right) \\
& \geq \frac{1}{2} u_{\lambda}^{\prime}(T)^{2} \geq C_{\varepsilon} \lambda e^{-2 \delta_{\lambda, \varepsilon} \sqrt{\lambda}} .
\end{aligned}
$$

Consequently, by (3.27)-(3.29), we obtain (3.25). Thus the proof is complete.

Lemma 3.10. Assume (A.1)-(A.4). Let $2 \pi<\alpha<4 \pi$ and $0<\varepsilon \ll 1$ be fixed. Assume that $2 \pi+\varepsilon \leq\left\|u_{\lambda}\right\|_{\infty}<4 \pi$ for $\lambda \gg 1$. Suppose that there exists a subsequence of $\left\{\lambda_{j}\right\}_{j=1}^{\infty}$ such that $\lambda_{j} \rightarrow \infty$ as $j \rightarrow \infty$ and satisfies (3.24) for $j \in \mathbb{N}$. Then for $j \gg 1$

$$
l_{\lambda_{j}, \varepsilon}=t_{2 \pi, \lambda_{j}}-t_{2 \pi+\varepsilon, \lambda_{j}} \leq \delta_{\lambda_{j}, \varepsilon}+O(\varepsilon)+o(1) .
$$

Proof. Since (3.24) is assumed, we have (3.10) and (3.29). By (3.10) and (3.29), we obtain (3.30).

Next, we estimate $t_{4 \pi-\varepsilon, \lambda}$ by $\delta_{\lambda, \varepsilon}$ from below in Lemma 3.12. To do this, we use the following Lemma 3.11.

Lemma 3.11. Assume (A.1)-(A.4). Let $\alpha>0$ be fixed. Suppose that $\sigma_{\lambda}:=$ $4 \pi-\left\|u_{\lambda}\right\|_{\infty} \rightarrow 0$ as $\lambda \rightarrow \infty$. Then

$$
\sigma_{\lambda}^{2} \leq C \frac{\mu(\lambda)}{\lambda} \text { for } \lambda \gg 1
$$

In particular, for $2 \pi<\alpha<4 \pi$, if (A.1)-(A.4), (A.5.1) and (1.5) for $n=1$ are assumed, then $\left\|u_{\lambda}\right\|_{\infty} \rightarrow 4 \pi$ as $\lambda \rightarrow \infty$, namely, $\sigma_{\lambda} \rightarrow 0$ as $\lambda \rightarrow \infty$. Furthermore, (3.31) holds.

Proof. Since $\sigma_{\lambda} \rightarrow 0$ as $\lambda \rightarrow \infty$, we see that $\left\|u_{\lambda}\right\|_{\infty} \leq C$. Then by (2.4), for $\lambda \gg 1$, we obtain

$$
\mu(\lambda) F(C) \geq \mu F\left(\left\|u_{\lambda}\right\|_{\infty}\right)=\frac{1}{2} u_{\lambda}^{\prime}(T)^{2}+\lambda\left(1-\cos \sigma_{\lambda}\right) \geq \frac{\lambda \sigma_{\lambda}^{2}}{4} .
$$

This implies (3.31). If we assume (A.1)-(A.4), (A.5.1) and (1.5) for $n=1$, then by Lemma 3.8, we have $\sigma_{\lambda}>0$ for $\lambda \gg 1$. Further, $\sigma_{\lambda} \rightarrow 0$ as $\lambda \rightarrow \infty$. Indeed, if there exists a subsequence of $\{\lambda\}$, denoted by $\{\lambda\}$ again, such that $\sigma_{\lambda} \geq C$, then by (2.7), we see that $u_{\lambda} \rightarrow 2 \pi$ or $u_{\lambda} \rightarrow 0$ a.e. in $I$ as $\lambda \rightarrow \infty$. Then

$$
2 T F(\alpha)=\int_{I} F\left(u_{\lambda}(t)\right) d t \leq 2 T F(2 \pi)+o(1) \quad \text { for } \lambda \gg 1 .
$$

This contradicts $\alpha>2 \pi$. Hence, we also obtain (3.31) in this case. 
Lemma 3.12. Assume (A.1)-(A.4). Let $\alpha>0$ and $0<\varepsilon \ll 1$ be fixed. Assume that $\|u\|_{\infty}<4 \pi$ and $\|u\|_{\infty} \rightarrow 4 \pi$ as $\lambda \rightarrow \infty$. Then for $\lambda \gg 1$

$$
t_{4 \pi-\varepsilon, \lambda} \geq \sqrt{1-2 \varepsilon} \delta_{\lambda, \varepsilon}-o(1) .
$$

Proof. Since $\left\|u_{\lambda}\right\|_{\infty} \rightarrow 4 \pi$, we see that $t_{4 \pi-\varepsilon, \lambda}$ exists for $\lambda \gg 1$. By (2.4), for $t \in\left[0, t_{4 \pi-\varepsilon, \lambda}\right]$,

$$
\begin{aligned}
\frac{1}{2} u_{\lambda}^{\prime}(t)^{2} & =\frac{1}{2} u_{\lambda}^{\prime}(T)^{2}+\lambda\left(1-\cos u_{\lambda}(t)\right)-\mu(\lambda) F\left(u_{\lambda}(t)\right) \\
& \leq \frac{1}{2} u_{\lambda}^{\prime}(T)^{2}+\lambda\left(1-\cos \left(4 \pi-u_{\lambda}(t)\right)\right) \\
& \leq \frac{1}{2} u_{\lambda}^{\prime}(T)^{2}+\frac{1}{2}\left(4 \pi-u_{\lambda}(t)\right)^{2} .
\end{aligned}
$$

By this, we obtain

$$
\begin{aligned}
t_{4 \pi-\varepsilon, \lambda}= & \int_{0}^{t_{4 \pi-\varepsilon, \lambda}} 1 d t \geq \int_{0}^{t_{4 \pi-\varepsilon, \lambda}} \frac{-u_{\lambda}^{\prime}(t)}{\sqrt{u_{\lambda}^{\prime}(T)^{2}+\lambda\left(4 \pi-u_{\lambda}(t)\right)^{2}}} d t \\
= & \int_{\sigma_{\lambda}}^{\varepsilon} \frac{1}{\sqrt{u_{\lambda}^{\prime}(T)^{2}+\lambda s^{2}}} d s \\
= & \frac{1}{\sqrt{\lambda}}\left[\log \left(\varepsilon+\sqrt{\varepsilon^{2}+u_{\lambda}^{\prime}(T)^{2} / \lambda}\right)\right. \\
& \left.-\log \left(\sigma_{\lambda}+\sqrt{\sigma_{\lambda}^{2}+u_{\lambda}^{\prime}(T)^{2} / \lambda}\right)\right] .
\end{aligned}
$$

By (2.4), we have

(3.36) $\frac{u_{\lambda}^{\prime}(T)^{2}}{\lambda} \leq \frac{u_{\lambda}^{\prime}(T)^{2}}{\lambda}+2\left(1-\cos \left\|u_{\lambda}\right\|_{\infty}\right)=\frac{2 F\left(\left\|u_{\lambda}\right\|_{\infty}\right) \mu(\lambda)}{\lambda} \leq \frac{2 F(4 \pi) \mu(\lambda)}{\lambda}$.

By this, Lemma 3.11 and (3.35), we have

$$
t_{4 \pi-\varepsilon, \lambda} \geq \frac{1}{\sqrt{\lambda}}\left(\log 2 \varepsilon+\log \left(\frac{\lambda}{\mu(\lambda)}\right)^{1 / 2}-\log C\right) .
$$

Since $\mu(\lambda) F(2 \pi) \leq u_{\lambda}^{\prime}(T)^{2} / 2$ by (3.4), by this and Lemma 2.6 , we obtain

$$
C e^{2 \delta_{\lambda, \varepsilon} \sqrt{(1-2 \varepsilon) \lambda}} \leq \frac{\lambda}{\mu(\lambda)} .
$$

By this and (3.37), we obtain (3.34).

Now we estimate $t_{4 \pi-\varepsilon, \lambda}$ by $\delta_{\lambda, \varepsilon}$ from above. To do this, we define $Q_{\lambda}$ by

$$
Q_{\lambda}:=\frac{1}{2}\left\|u_{\lambda}^{\prime}\right\|_{2}^{2}-\lambda \int_{I}\left(1-\cos u_{\lambda}(t)\right) d t .
$$


Lemma 3.13. Assume (A.1)-(A.4), (A.5.1). Let $2 \pi<\alpha<4 \pi$ satisfy (1.5) for $n=1$. Then $Q_{\lambda} \leq 0$ for $\lambda \gg 1$.

Proof. Assume that there exists a subsequence of $\{\lambda\}$, which is denoted by $\{\lambda\}$ again, such that $\lambda \rightarrow \infty$ and $Q_{\lambda}>0$. Integrate (3.4) over $I$ to obtain

$Q_{\lambda}=T u_{\lambda}^{\prime}\left(t_{2 \pi, \lambda}\right)^{2}-2 T \mu(\lambda)(F(\alpha)-F(2 \pi))=T u_{\lambda}^{\prime}(T)^{2}-2 T \mu(\lambda) F(\alpha)$.

Since we assume $Q_{\lambda}>0$ for $\lambda \gg 1$, we see from this that for $\lambda \gg 1$

$$
T u_{\lambda}^{\prime}\left(t_{2 \pi, \lambda}\right)^{2}>2 T \mu(\lambda)(F(\alpha)-F(2 \pi)) .
$$

This implies (3.24). Then by Lemmas 2.7, 3.6, 3.9 and 3.10, for $\lambda \gg 1$, we have

$$
m_{\lambda, \varepsilon}=\delta_{\lambda, \varepsilon}+O(\varepsilon)+o(1), \quad l_{\lambda, \varepsilon}=\delta_{\lambda, \varepsilon}+O(\varepsilon)+o(1) .
$$

This along with (3.19) and Lemma 3.12 implies that

$$
T=t_{4 \pi-\varepsilon, \lambda}+3 \delta_{\lambda, \varepsilon}+O(\varepsilon)+o(1) \geq 4 \delta_{\lambda, \varepsilon}-C \varepsilon-o(1) .
$$

Then by this, (3.42) and Lemma 3.12, for $\lambda \gg 1$, we obtain

$$
\begin{aligned}
T F(\alpha) & =F(4 \pi) t_{4 \pi-\varepsilon, \lambda}+2 F(2 \pi) \delta_{\lambda, \varepsilon}+O(\varepsilon)+o(1) \\
& \geq F(4 \pi)\left(T-3 \delta_{\lambda, \varepsilon}\right)+2 F(2 \pi) \delta_{\lambda, \varepsilon}-C \varepsilon-o(1) \\
& =T F(4 \pi)+(2 F(2 \pi)-3 F(4 \pi)) \delta_{\lambda, \varepsilon}-C \varepsilon-o(1) \\
& \geq T F(4 \pi)+(2 F(2 \pi)-3 F(4 \pi)) T / 4-C \varepsilon-o(1) \\
& =\frac{T}{4} F(4 \pi)+\frac{T}{2} F(2 \pi)-C \varepsilon-o(1) .
\end{aligned}
$$

This contradicts (1.5) for $n=1$. Thus the proof is complete.

Lemma 3.14. Assume (A.1)-(A.4). Let $2 \pi<\alpha<4 \pi$ be fixed. If $\left\|u_{\lambda}\right\|_{\infty} \rightarrow$ $4 \pi$ as $\lambda \rightarrow \infty$ and $Q_{\lambda} \leq 0$ for $\lambda \gg 1$, then there exists a constant $C>0$ such that for $\lambda \gg 1$

$$
\frac{\mu(\lambda)}{\lambda} \leq C \sigma_{\lambda}^{2}
$$

In particular, if (A.1)-(A.4) and (A.5.1) are fulfilled and $2 \pi<\alpha<4 \pi$ satisfies (1.5) for $n=1$, then (3.43) holds.

Proof. Since $Q_{\lambda} \leq 0$, by (3.40), we have

$$
\frac{1}{2} u_{\lambda}^{\prime}(T)^{2} \leq \mu(\lambda) F(\alpha) .
$$

Then by this and (2.4), we obtain

$$
\begin{aligned}
\frac{1}{2} \lambda \sigma_{\lambda}^{2} & \geq \lambda\left(1-\cos \left\|u_{\lambda}\right\|_{\infty}\right)=\mu(\lambda) F\left(\left\|u_{\lambda}\right\|_{\infty}\right)-\frac{1}{2} u_{\lambda}^{\prime}(T)^{2} \\
& \geq \mu(\lambda)\left(F\left(\left\|u_{\lambda}\right\|_{\infty}\right)-F(\alpha)\right) .
\end{aligned}
$$


This implies (3.43). If $2 \pi<\alpha<4 \pi$ satisfies (1.5) for $n=1$, then by Lemmas 3.8, 3.11 and 3.13, the assumptions in this lemma are satisfied. Hence we obtain (3.43).

Lemma 3.15. Assume (A.1)-(A.4). Let $2 \pi<\alpha<4 \pi$ and $0<\varepsilon \ll 1$ be fixed. If $\left\|u_{\lambda}\right\|_{\infty}<4 \pi,\left\|u_{\lambda}\right\|_{\infty} \rightarrow 4 \pi$ as $\lambda \rightarrow \infty$ and $Q_{\lambda} \leq 0$ for $\lambda \gg 1$, then for $\lambda \gg 1$

$$
t_{4 \pi-\varepsilon, \lambda} \leq \delta_{\lambda, \varepsilon}+O(\varepsilon)+o(1)
$$

In particular, if (A.1)-(A.4) and (A.5.1) are fulfilled and $2 \pi<\alpha<4 \pi$ satisfies (1.5) for $n=1$, then (3.46) holds.

Proof. We see that for $4 \pi-\varepsilon \leq u \leq 4 \pi-\sigma_{\lambda}$

$$
\left(\left\|u_{\lambda}\right\|_{\infty}-u\right) \sin \sigma_{\lambda}+\frac{1-C \varepsilon}{2}\left(u-\left\|u_{\lambda}\right\|_{\infty}\right)^{2} \leq \cos \left\|u_{\lambda}\right\|_{\infty}-\cos u .
$$

Indeed, (3.47) is equvalent to

$$
g(\theta)=\cos \sigma_{\lambda}-\cos \left(\theta+\sigma_{\lambda}\right)-\theta \sin \sigma_{\lambda}-(1-C \varepsilon) \theta^{2} / 2 \geq 0
$$

for $0 \leq \theta \leq \varepsilon-\sigma_{\lambda}$. Then it is easy to see that $g(0)=g^{\prime}(0)=0$ and $g^{\prime \prime}(\theta)>0$ for $0 \leq \theta \leq \varepsilon-\sigma_{\lambda}$. Hence $g(\theta) \geq 0$ for $0 \leq \theta \leq \varepsilon-\sigma_{\lambda}$, and we obtain (3.47). Then by (2.4) and the inequality $\sin \sigma_{\lambda} \geq(1-C \varepsilon) \sigma_{\lambda} / 2$, for $t \in\left[0, t_{4 \pi-\varepsilon, \lambda}\right]$ and $\lambda \gg 1$, we obtain

$$
\begin{aligned}
\frac{1}{2} u_{\lambda}^{\prime}(t)^{2} & =\lambda\left(\cos \left\|u_{\lambda}\right\|_{\infty}-\cos u_{\lambda}(t)\right)+\mu(\lambda)\left(F\left(\left\|u_{\lambda}\right\|_{\infty}\right)-F\left(u_{\lambda}(t)\right)\right. \\
& \geq \lambda \sin \sigma_{\lambda}\left(\left\|u_{\lambda}\right\|_{\infty}-u_{\lambda}(t)\right)+\frac{1-C \varepsilon}{2} \lambda\left(\left\|u_{\lambda}\right\|_{\infty}-u_{\lambda}(t)\right)^{2} \\
& \geq \frac{(1-C \varepsilon)}{2} \lambda \sigma_{\lambda}\left(\left\|u_{\lambda}\right\|_{\infty}-u_{\lambda}(t)\right)+\frac{1-C \varepsilon}{2} \lambda\left(\left\|u_{\lambda}\right\|_{\infty}-u_{\lambda}(t)\right)^{2} .
\end{aligned}
$$

This implies

$$
\begin{aligned}
t_{4 \pi-\varepsilon, \lambda} \leq & \int_{0}^{t_{4 \pi-\varepsilon, \lambda}} 1 d t=\frac{1}{\sqrt{(1-C \varepsilon) \lambda}} \\
& \cdot \int_{0}^{t_{4 \pi-\varepsilon, \lambda}} \frac{-u_{\lambda}^{\prime}(t)}{\sqrt{\left(\left\|u_{\lambda}\right\|_{\infty}-u_{\lambda}(t)\right)^{2}+\sigma_{\lambda}\left(\left\|u_{\lambda}\right\|_{\infty}-u_{\lambda}(t)\right)}} d t \\
= & \frac{1}{\sqrt{(1-C \varepsilon) \lambda}} \int_{0}^{\varepsilon-\sigma_{\lambda}} \frac{1}{\sqrt{s^{2}+\sigma_{\lambda} s}} d s \\
< & \frac{1}{\sqrt{(1-C \varepsilon) \lambda}} \lim _{\zeta \rightarrow 0} \int_{\zeta}^{\varepsilon} \frac{1}{\sqrt{s^{2}+\sigma_{\lambda} s}} d s
\end{aligned}
$$




$$
\begin{aligned}
& =\lim _{\zeta \rightarrow 0} \frac{1}{\sqrt{(1-C \varepsilon) \lambda}}\left[\log \left|\frac{t+1}{t-1}\right|\right]_{\sqrt{\left(\zeta+\sigma_{\lambda}\right) / \zeta}}^{\sqrt{\left(\varepsilon+\sigma_{\lambda}\right) / \varepsilon}} \\
& =\frac{1}{\sqrt{(1-C \varepsilon) \lambda}} \log \left|\frac{\sqrt{\left(\varepsilon+\sigma_{\lambda}\right) / \varepsilon}+1}{\sqrt{\left(\varepsilon+\sigma_{\lambda}\right) / \varepsilon}-1}\right| .
\end{aligned}
$$

We easily see that $\sqrt{\left(\varepsilon+\sigma_{\lambda}\right) / \varepsilon} \geq 1+C_{\varepsilon} \sigma_{\lambda}$ for some constant $C_{\varepsilon}>0$. Consequently, by (3.48) and Lemma 3.14, we have

$$
\begin{aligned}
t_{4 \pi-\varepsilon, \lambda} & \leq \frac{1}{\sqrt{(1-C \varepsilon) \lambda}}\left(\log \sigma_{\lambda}^{-1}+C\right) \\
& \leq \frac{1}{\sqrt{(1-C \varepsilon) \lambda}}\left\{\log \left(\frac{\lambda}{\mu(\lambda)}\right)^{1 / 2}+C\right\} .
\end{aligned}
$$

By Lemma 3.1 and (3.44), we have $\lambda / \mu(\lambda) \leq C e^{2 \delta_{\lambda, \varepsilon} \sqrt{\lambda}}$. By this and (3.49), we obtain (3.46). Finally, if $2 \pi<\alpha<4 \pi$ satisfies (1.5) for $n=1$, then by Lemmas 3.8, 3.11 and 3.13 , we see that the assumptions in this lemma are satisfied. Therefore, we obtain (3.46). Thus the proof is complete.

Proof of TheOrem 2(ii)-(vi) For $n=1$. Let an arbitrary $0<\varepsilon \ll 1$ be fixed. Then by Lemmas 3.12 and 3.15, we see that for $\lambda \gg 1$

$$
t_{4 \pi-\varepsilon, \lambda}=\delta_{\lambda, \varepsilon}+O(\varepsilon)+o(1)
$$

By (3.19) and (3.50), for $\lambda \gg 1$, we obtain

$$
\begin{aligned}
T F(\alpha) & =F(4 \pi) t_{4 \pi-\varepsilon, \lambda}+\left(T-t_{4 \pi-\varepsilon, \lambda}-\delta_{\lambda, \varepsilon}\right) F(2 \pi)+O(\varepsilon)+o(1) \\
& =T F(2 \pi)+\delta_{\lambda, \varepsilon}(F(4 \pi)-2 F(2 \pi))+O(\varepsilon)+o(1) .
\end{aligned}
$$

This implies that for $\lambda \gg 1$,

$$
\delta_{\lambda, \varepsilon}=\frac{F(\alpha)-F(2 \pi)}{F(4 \pi)-2 F(2 \pi)} T+O(\varepsilon)+o(1)=T_{\alpha, 1}+O(\varepsilon)+o(1) .
$$

Now Theorem 2(ii)-(v) for $n=1$ are direct consequence of (3.50) and (3.51). Finally, (1.6) follows from (3.38) and (3.51).

Proof of TheOrem 2 FOR $n \geq 2$. For $n \geq 2$, we can prove Theorem 2 as follows. Since $2 n \pi<\alpha<2(n+1) \pi$, we have $\left\|u_{\lambda}\right\|_{\infty}>2 n \pi$. By using (A.5. $n$ ) and the same argument as that in Lemma 3.8, we first obtain $\left\|u_{\lambda}\right\|_{\infty}<2(n+1) \pi$. Secondly, let an arbitrary $0<\varepsilon \ll 1$ be fixed. For $1 \leq k \leq n$, we put

$$
l_{\lambda, \varepsilon, k}:=t_{2 k \pi, \lambda}-t_{2 k \pi+\varepsilon, \lambda}, \quad m_{\lambda, \varepsilon, k}:=t_{2 k \pi-\varepsilon, \lambda}-t_{2 k \pi, \lambda} .
$$

Then by replacing $2 \pi$ with $2 k \pi$, we repeat the same calculation as those of Lemmas 2.7 and 3.6. Then for $1 \leq k \leq n-1$ and $\lambda \gg 1$, we obtain

$$
l_{\lambda, \varepsilon, k}, \quad m_{\lambda, \varepsilon, k} \geq \delta_{\lambda, \varepsilon}-C \varepsilon-o(1) .
$$


Since $\left\|u_{\lambda}\right\|_{\infty}>2 n \pi$, there exists $t_{2 k \pi, \lambda}$ for $1 \leq k \leq n$. Then by putting $t=2 k \pi$ in (2.4), we obtain that for $1 \leq k \leq n-1$

$\frac{1}{2} u_{\lambda}^{\prime}\left(t_{2 n \pi, \lambda}\right)^{2}+\mu(\lambda) F(2 n \pi)+\lambda=\frac{1}{2} u_{\lambda}^{\prime}\left(t_{2 k \pi, \lambda}\right)^{2}+\mu(\lambda) F(2 k \pi)+\lambda=\frac{1}{2} u_{\lambda}^{\prime}(T)^{2}+\lambda$.

This implies that for $1 \leq k \leq n-1$

$$
\frac{1}{2} u_{\lambda}^{\prime}(T)^{2} \geq \frac{1}{2} u_{\lambda}^{\prime}\left(t_{2 k \pi, \lambda}\right)^{2} \geq \mu(\lambda)(F(2 n \pi)-F(2 k \pi))
$$

(3.54) corresponds with (3.24) for $1 \leq k \leq n-1$. Then by repeating the same arguments as those of Lemmas 3.9 and 3.10, for $1 \leq k \leq n-1$ and $\lambda \gg 1$, we obtain

$$
l_{\lambda, \varepsilon, k}, \quad m_{\lambda, \varepsilon, k} \leq \delta_{\lambda, \varepsilon}+O(\varepsilon)+o(1) .
$$

This along with (3.53) implies that for $1 \leq k \leq n-1$ and $\lambda \gg 1$

$$
l_{\lambda, \varepsilon, k}, \quad m_{\lambda, \varepsilon, k}=\delta_{\lambda, \varepsilon}+O(\varepsilon)+o(1) .
$$

Now by using the same arguments as those in Lemmas 3.11-3.15, for $\lambda \gg 1$, we obtain

$$
t_{2(n+1) \pi-\varepsilon, \lambda}=\delta_{\lambda, \varepsilon}+O(\varepsilon)+o(1) .
$$

By (2.7), we have

$$
T=t_{2(n+1) \pi-\varepsilon, \lambda}+\sum_{k=2}^{n}\left(l_{\lambda, \varepsilon, k}+m_{\lambda, \varepsilon, k}\right)+\left(l_{\lambda, \varepsilon}+m_{\lambda, \varepsilon}\right)+\delta_{\lambda, \varepsilon}+o(1) .
$$

Then by (3.55)-(3.57), we obtain

$$
\begin{aligned}
T F(\alpha)= & t_{2(n+1) \pi-\varepsilon, \lambda} F(2(n+1) \pi)+\left(T-t_{2(n+1) \pi-\varepsilon, \lambda}-(2 n-1) \delta_{\lambda, \varepsilon}\right) F(2 n \pi) \\
& +\sum_{k=0}^{n-1} 2 F(2 k \pi) \delta_{\lambda, \varepsilon}+O(\varepsilon)+o(1) \\
= & T F(2 n \pi)+\left\{F(2(n+1) \pi)-2 n F(2 n \pi)+2 \sum_{k=0}^{n-1} F(2 k \pi)\right\} \delta_{\lambda, \varepsilon} \\
& +O(\varepsilon)+o(1) \\
= & T F(2 n \pi)+H(n) \delta_{\lambda, \varepsilon}+O(\varepsilon)+o(1) .
\end{aligned}
$$

This implies that for $\lambda \gg 1$

$$
\delta_{\lambda, \varepsilon}=T_{\alpha, n}+O(\varepsilon)+o(1)
$$

Now Theorem 2(ii)-(v) are direct consequence of (3.55), (3.56) and (3.58). Finally, (1.6) follows from (3.54), (3.58) and Lemma 2.6. Thus the proof is complete. 


\section{Proof of Theorem 1}

Proof of Theorem 1 is a variant of the proof of Theorem 2.

Lemma 4.1. Assume (A.1)-(A.4) and (A.5.1). Assume that $0<\alpha<2 \pi$ satisfies $2 F(\alpha) \geq F(2 \pi)$. Then $\left\|u_{\lambda}\right\|_{\infty}<4 \pi$ for $\lambda \gg 1$.

Proof. Assume that there exists a subsequence of $\{\lambda\}$, which is denoted by $\{\lambda\}$ again, such that $\lambda \rightarrow \infty$ and $\left\|u_{\lambda}\right\|_{\infty} \geq 4 \pi$. Then by the same arguments as those in Lemmas 3.2-3.8, we obtain (3.23) for $0<\varepsilon \ll 1$. This implies

$$
F(2 \pi)>F(\alpha) \geq \frac{1}{4} F(4 \pi)+\frac{1}{2} F(2 \pi)-C \varepsilon-o(1) .
$$

Since $0<\varepsilon \ll 1$ is arbitrary, this implies that $2 F(2 \pi) \geq F(4 \pi)$. This contradicts (A.5.1).

By (2.4) and Lemma 4.1, we have

$$
\begin{aligned}
\lambda\left(1-\cos \left\|u_{\lambda}\right\|_{\infty}\right) & \leq \lambda\left(1-\cos \left\|u_{\lambda}\right\|_{\infty}\right)+\frac{1}{2} u_{\lambda}^{\prime}(T)^{2} \\
& =\mu(\lambda) F\left(\left\|u_{\lambda}\right\|_{\infty}\right)<\mu(\lambda) F(4 \pi) .
\end{aligned}
$$

By (4.1) and Lemmas 2.4 and 4.1, we have two possibilities: $\left\|u_{\lambda}\right\|_{\infty} \rightarrow 4 \pi$ or $\left\|u_{\lambda}\right\|_{\infty} \rightarrow 2 \pi$ as $\lambda \rightarrow \infty$.

Lemma 4.2. Assume (A.1)-(A.4) and (A.5.1). Assume that $0<\alpha<2 \pi$ satisfies $2 F(\alpha) \geq F(2 \pi)$. Then $\left\|u_{\lambda}\right\|_{\infty} \rightarrow 2 \pi$ as $\lambda \rightarrow \infty$.

Proof. Assume that there exists a subsequence of $\{\lambda\}$, which is denoted by $\{\lambda\}$ again, such that $\left\|u_{\lambda}\right\|_{\infty} \rightarrow 4 \pi$ as $\lambda \rightarrow \infty$. Let $0<\varepsilon \ll 1$ be fixed. By Lemma 3.12 and (3.19), we see that for $\lambda \gg 1$

$$
\begin{aligned}
T F(\alpha) & \geq t_{4 \pi-\varepsilon, \lambda} F(4 \pi-\varepsilon)+\left(T-t_{4 \pi-\varepsilon, \lambda}-\delta_{\lambda, \varepsilon}-o(1)\right) F(2 \pi-\varepsilon) \\
& \geq T F(2 \pi)+t_{4 \pi-\varepsilon, \lambda}(F(4 \pi)-2 F(2 \pi))-C \varepsilon-o(1) .
\end{aligned}
$$

This together with (A.5.1) contradicts the assumption $\alpha<2 \pi$. Thus the proof is complete.

By Lemma 4.2, we obtain

$$
T F(\alpha)=F(2 \pi) t_{2 \pi-\varepsilon, \lambda}+O(\varepsilon)+o(1)
$$

for $\lambda \gg 1$ and $0<\varepsilon \ll 1$. This implies $t_{2 \pi-\varepsilon, \lambda}=T_{\alpha, 0}+O(\varepsilon)+o(1)$. This implies the assertion (i) and (ii). The assertion (iii) is exactly the same as that of Theorem 0(iii). However, for completeness, the proof will be given in appendix. Thus the proof is complete. 


\section{Proof of Theorem 3}

We begin with the proof of Theorem 3(i) for $n=1$.

Proof of Theorem 3(i) FOR $n=1$. We assume that there exists a subsequence of $\{\lambda\}$, denoted by $\{\lambda\}$ again, such that $\lambda \rightarrow \infty$ and $\left\|u_{\lambda}\right\|_{\infty} \geq 6 \pi$ and derive a contradiction. We have the inequality (3.5), namely, (3.24) in this case. Therefore, for a fixed $0<\varepsilon \ll 1$, Lemma 3.9 and Lemma 3.10 are valid in this case. So these lemmas together with Lemmas 2.7 and 3.6 imply (3.42). Furthermore, by the same argument as that used in Lemma 2.7, we obtain that for $\lambda \gg 1$,

$$
t_{6 \pi-\varepsilon, \lambda} \geq t_{6 \pi-\varepsilon, \lambda}-t_{6 \pi, \lambda} \geq \sqrt{(1-2 \varepsilon)} \delta_{\lambda, \varepsilon}-o(1) .
$$

Then by (A.5.2), (3.42) and (5.1),

$$
\begin{aligned}
T F(\alpha) \geq & t_{6 \pi-\varepsilon, \lambda} F(6 \pi-\varepsilon)+\left(T-t_{6 \pi-\varepsilon, \lambda}-m_{\lambda, \varepsilon}-l_{\lambda, \varepsilon}-\delta_{\lambda, \varepsilon}\right) F(4 \pi-\varepsilon) \\
& +\left(m_{\lambda, \varepsilon}+l_{\lambda, \varepsilon}\right) F(2 \pi-\varepsilon)-o(1) \\
\geq & T F(4 \pi)+t_{6 \pi-\varepsilon, \lambda}(F(6 \pi)-F(4 \pi))-3 \delta_{\lambda, \varepsilon} F(4 \pi) \\
& \quad+2 \delta_{\lambda, \varepsilon} F(2 \pi)-C \varepsilon-o(1) \\
\geq & T F(4 \pi)+\delta_{\lambda, \varepsilon}(F(6 \pi)-4 F(4 \pi)+2 F(2 \pi))-C \varepsilon-o(1) \\
> & T F(4 \pi)-C \varepsilon-o(1) .
\end{aligned}
$$

Since $0<\varepsilon \ll 1$ is arbitrary, this contradicts the assumption $\alpha<4 \pi$. Thus we obtain $\left\|u_{\lambda}\right\|_{\infty}<6 \pi$. If there exists a subsequence of $\{\lambda\}$, denoted by $\{\lambda\}$ again, such that $\left\|u_{\lambda}\right\|_{\infty} \rightarrow 6 \pi$, then by the same calculation as that of Lemma 3.12, for $\lambda \gg 1$, we obtain $t_{6 \pi-\varepsilon, \lambda} \geq \sqrt{(1-2 \varepsilon)} \delta_{\lambda, \varepsilon}-o(1)$. By using this and the same argument as that of (5.2), we can also derive a contradiction in this case. Thus we obtain that $\left\|u_{\lambda}\right\|_{\infty} \rightarrow 4 \pi$ as $\lambda \rightarrow \infty$.

Now we are ready to prove Theorem $3(\mathrm{ii})-(\mathrm{v})$ for $n=1$.

Proof of TheOrem 3(ii)-(v) For $n=1$. We first consider the case where $F(\alpha)>F(4 \pi) / 4+F(2 \pi) / 2$. Then there are two cases to consider.

Case 1. Assume that there exists a subsequence of $\{\lambda\}$, denoted by $\{\lambda\}$ again, such that $\lambda \rightarrow \infty$ and $\left\|u_{\lambda}\right\|_{\infty}<4 \pi$. We first prove that $Q_{\lambda}>0$ for $\lambda \gg 1$, where $Q_{\lambda}$ is defined in (3.39). Assume, on the contrary, that there exists a subsequence of $\{\lambda\}$ such that $\lambda \rightarrow \infty$ and $Q_{\lambda} \leq 0$. Let $0<\varepsilon \ll 1$ be fixed. Then by Lemmas 3.12 and 3.15, for $\lambda \gg 1$, we obtain (3.50). Then by (3.19), (3.50), Lemmas 2.7 and 3.6, for $\lambda \gg 1$, we obtain

$$
T=t_{4 \pi-\varepsilon, \lambda}+m_{\lambda, \varepsilon}+l_{\lambda, \varepsilon}+\delta_{\lambda, \varepsilon}+o(1) \geq 4 \delta_{\lambda, \varepsilon}-C \varepsilon-o(1) .
$$


Then by (3.50) and (5.3)

$$
\begin{aligned}
T F(\alpha) & =F(4 \pi) t_{4 \pi-\varepsilon, \lambda}+F(2 \pi)\left(T-t_{4 \pi-\varepsilon, \lambda}-\delta_{\lambda, \varepsilon}\right)+O(\varepsilon)+o(1) \\
& =T F(2 \pi)+\delta_{\lambda, \varepsilon}(F(4 \pi)-2 F(2 \pi))+O(\varepsilon)+o(1) \\
& \leq T F(2 \pi)+\frac{T}{4}(F(4 \pi)-2 F(2 \pi))+O(\varepsilon)+o(1) \\
& =\frac{T}{4} F(4 \pi)+\frac{T}{2} F(2 \pi)+O(\varepsilon)+o(1) .
\end{aligned}
$$

This is a contradiction. Thus we obtain that $Q_{\lambda}>0$ for $\lambda \gg 1$, which implies (3.24) by (3.41). Then by (3.24), Lemmas 2.7, 3.6, 3.9 and 3.10, we obtain (3.42). This implies that

$$
T F(\alpha)=\left(T-3 \delta_{\lambda, \varepsilon}\right) F(4 \pi)+2 \delta_{\lambda, \varepsilon} F(2 \pi)+O(\varepsilon)+o(1) .
$$

Hence we see that $\delta_{\lambda, \varepsilon}=S_{\alpha, 1}+O(\varepsilon)+o(1)$ for $\lambda \gg 1$. This along with (3.42) implies Theorem 3(ii)-(iv). Theorem 3(v) follows from (3.38), Lemma 2.6 and the fact that $\delta_{\lambda, \varepsilon}=S_{\alpha, 1}+O(\varepsilon)+o(1)$ for $\lambda \gg 1$. Thus the proof of Case 1 is complete.

Case 2. Assume that there exists a subsequence of $\{\lambda\}$, denoted by $\{\lambda\}$ again, such that $\lambda \rightarrow \infty$ and $\left\|u_{\lambda}\right\|_{\infty} \geq 4 \pi$. Then by (3.4), we obtain (3.5), which implies (3.24). Hence, we find that Lemma 3.9 and Lemma 3.10 are valid in this case. Namely, we have (3.42). Then by the same argument as that in Case 1, we also obtain (5.5), which implies Theorem 3(ii)-(iv) in this case. Finally, Theorem 3(v) follows from (3.38), Lemma 2.6, and the fact that $\delta_{\lambda, \varepsilon}=S_{\alpha, 1}+O(\varepsilon)+o(1)$ for $\lambda \gg 1$. Thus the proof of the case $n=1$ is complete.

Now, we consider the case where $F(\alpha)=F(4 \pi) / 4+F(2 \pi) / 2$. There are two cases to consider.

Case 3. Assume that there exists a subsequence of $\{\lambda\}$, denoted by $\{\lambda\}$ again, such that $\lambda \rightarrow \infty$ and $\left\|u_{\lambda}\right\|_{\infty} \geq 4 \pi$. Let $0<\varepsilon \ll 1$ be fixed. Then by (3.5), we see that Lemma 3.9 and Lemma 3.10 are valid. By these facts and Lemmas 2.7 and 3.6, we obtain (3.42), which implies

$$
T F(\alpha)=\frac{T}{4} F(4 \pi)+\frac{T}{2} F(2 \pi)=\left(T-3 \delta_{\lambda, \varepsilon}\right) F(4 \pi)+2 \delta_{\lambda, \varepsilon} F(2 \pi)+O(\varepsilon)+o(1) .
$$

Hence for $\lambda \gg 1$, we obtain

$$
\delta_{\lambda, \varepsilon}=T / 4+O(\varepsilon)+o(1) .
$$

This implies Theorem 3(iv). The assertions (ii) and (iii) follow from (3.42) and (5.6). The assertion (v) follows from (3.38), (5.6) and Lemma 2.6.

Case 4. Assume that there exists a subsequence of $\{\lambda\}$, denoted by $\{\lambda\}$ again, such that $\lambda \rightarrow \infty$ and $\left\|u_{\lambda}\right\|_{\infty}<4 \pi$. If there exists a subsequence of $\{\lambda\}$ such that $Q_{\lambda}>0$, then our conclusion follows exactly from the same argument 
as that of Case 3. If there exists a subsequence of $\{\lambda\}$ such that $Q_{\lambda} \leq 0$, then by Lemmas 3.12 and 3.15, we have (3.50). Then

$$
T F(\alpha)=\frac{T}{4} F(4 \pi)+\frac{T}{2} F(2 \pi)=\delta_{\lambda, \varepsilon} F(4 \pi)+\left(T-2 \delta_{\lambda, \varepsilon}\right) F(2 \pi)+O(\varepsilon)+o(1) .
$$

This implies (5.6). Then by the same argument as that in Case 3, we obtain our conclusion. Thus the proof for the case $n=1$ is complete.

Proof of TheOrem 3 For $n \geq 2$. Let $0<\varepsilon \ll 1$ be fixed. We recall $l_{\lambda, \varepsilon, k}, m_{\lambda, \varepsilon, k}$ defined in (3.52) for $1 \leq k \leq n$. Then by the same arguments as those in the proof of Theorem 2 for $n \geq 2$, for $1 \leq k \leq n-1$, we obtain (3.55). Then by the same argument as that of the proof of Theorem 3 for $n=1$, we obtain $\left\|u_{\lambda}\right\|_{\infty} \rightarrow 2(n+1) \pi$ as $\lambda \rightarrow \infty$. This implies Theorem 3(i). By the same calculation as those for the case $n=1$, we obtain

$$
l_{\lambda, \varepsilon, n}, m_{\lambda, \varepsilon, n}=\delta_{\lambda, \varepsilon}+O(\varepsilon)+o(1) .
$$

Therefore, by (3.55) and (5.7), we obtain

$$
T F(\alpha)=\left(T-(2 n+1) \delta_{\lambda, \varepsilon}\right) F(2(n+1) \pi)+2 \delta_{\lambda, \varepsilon} \sum_{k=0}^{n} F(2 k \pi)+O(\varepsilon)+o(1) .
$$

This implies that for $\lambda \gg 1$

$$
\delta_{\lambda, \varepsilon}=S_{\alpha, n}+O(\varepsilon)+o(1)
$$

This implies Theorem 3(ii)-(iv). Finally, Theorem 3(v) follows from (3.38), (5.8) and Lemma 2.6.

\section{Proof of Theorem 4}

We first prove Theorem 4 for $n=1$.

Lemma 6.1. Assume (A.1)-(A.4) and (A.5.1). Let $\alpha=2 \pi$. Then $\left\|u_{\lambda}\right\|_{\infty}<$ $4 \pi$ for $\lambda \gg 1$.

Proof. We note that $\left\|u_{\lambda}\right\|_{\infty} \geq 2 \pi$, since $u_{\lambda} \in M_{2 \pi}$. Assume that there exists a subsequence of $\{\lambda\}$, denoted by $\{\lambda\}$ again, such that $\lambda \rightarrow \infty$ and $\left\|u_{\lambda}\right\|_{\infty} \geq 4 \pi$. Let $0<\varepsilon \ll 1$ be fixed. Then by Lemmas $2.7,3.3,3.5,3.6$, we have

$$
\begin{aligned}
t_{4 \pi-\varepsilon, \lambda}>t_{4 \pi-\varepsilon, \lambda}-t_{4 \pi, \lambda} & \geq \frac{m_{\lambda, \varepsilon}+l_{\lambda, \varepsilon}}{2}-C \varepsilon-o(1), \\
\frac{m_{\lambda, \varepsilon}+l_{\lambda, \varepsilon}}{2} & \geq \delta_{\lambda, \varepsilon}-C \varepsilon-o(1) .
\end{aligned}
$$

By (6.1) and (6.2), we obtain

$$
\begin{aligned}
T F(2 \pi) & \geq t_{4 \pi-\varepsilon, \lambda} F(4 \pi-\varepsilon)+\left(T-t_{4 \pi-\varepsilon, \lambda}-\delta_{\lambda, \varepsilon}\right) F(2 \pi-\varepsilon) \\
& \geq T F(2 \pi)+t_{4 \pi-\varepsilon, \lambda}(F(4 \pi)-2 F(2 \pi))-C \varepsilon-o(1) .
\end{aligned}
$$


This along with (A.5.1), (6.1) and (6.2) implies that for $\lambda \gg 1$

$$
t_{4 \pi-\varepsilon, \lambda} \leq C \varepsilon+o(1), \quad \delta_{\lambda, \varepsilon} \leq C \varepsilon+o(1) .
$$

By (3.19) and (6.3), for $\lambda \gg 1$, we obtain

$$
l_{\lambda, \varepsilon}+m_{\lambda, \varepsilon} \geq T-C \varepsilon-o(1) .
$$

This along with (6.1) implies that for $\lambda \gg 1$

$$
t_{4 \pi-\varepsilon, \lambda} \geq T / 2-C \varepsilon-o(1) .
$$

This contradicts (6.3). Thus, we obtain $\left\|u_{\lambda}\right\|_{\infty}<4 \pi$ for $\lambda \gg 1$.

By Lemma 6.1, we have (4.1) in this case. So there are only two possibilities: $\left\|u_{\lambda}\right\|_{\infty} \rightarrow 4 \pi$ or $\left\|u_{\lambda}\right\|_{\infty} \rightarrow 2 \pi$ as $\lambda \rightarrow \infty$. If $\left\|u_{\lambda}\right\|_{\infty} \rightarrow 2 \pi$ as $\lambda \rightarrow \infty$, then $u_{\lambda} \rightarrow$ $2 \pi$ as $\lambda \rightarrow \infty$ locally uniformly on $(0, T)$, since $u_{\lambda} \in M_{2 \pi}$. Hence Theorem 4 is obtained immediately in this case.

Lemma 6.2. Assume (A.1)-(A.4) and (A.5.1) and $\alpha=2 \pi$. Suppose that $\left\|u_{\lambda}\right\|_{\infty} \rightarrow 4 \pi$ as $\lambda \rightarrow \infty$. Let $0<\varepsilon \ll 1$ be fixed. Then (6.4) holds for $\lambda \gg 1$.

Proof. By Lemma 3.12 and (3.19), we obtain

$$
\begin{aligned}
T F(2 \pi) & \geq t_{4 \pi-\varepsilon, \lambda} F(4 \pi)+\left(T-t_{4 \pi-\varepsilon, \lambda}-\delta_{\lambda, \varepsilon}\right) F(2 \pi)-C \varepsilon-o(1) \\
& =T F(2 \pi)+t_{4 \pi-\varepsilon, \lambda}(F(4 \pi)-2 F(2 \pi))-C \varepsilon-o(1) .
\end{aligned}
$$

This along with (A.5.1) and Lemma 3.12 implies (6.3). Then by (3.19) and (6.3), we obtain (6.4). Thus the proof is complete.

(6.4) implies that $u_{\lambda} \rightarrow 2 \pi$ on any compact subset in $(0, T)$. Now we obtain Theorem 4 for $n=1$ by Lemmas 6.1-6.2.

Proof of Theorem 4 For $n \geq 2$. Let $\alpha=2 n \pi$ for $n \geq 2$. We first prove $\left\|u_{\lambda}\right\|_{\infty}<2(n+1) \pi$ for $\lambda \gg 1$. To this end, we assume that there exists a subsequence of $\{\lambda\}$, denoted by $\{\lambda\}$ again, such that $\left\|u_{\lambda}\right\|_{\infty} \geq 2(n+1) \pi$ for $\lambda \gg 1$ and derive a contradiction. Let $0<\varepsilon \ll 1$ be fixed. By the same argument as that of Lemma 2.7, for $\lambda \gg 1$, we obtain

$$
t_{2(n+1) \pi-\varepsilon, \lambda} \geq t_{2(n+1) \pi-\varepsilon, \lambda}-t_{2(n+1) \pi, \lambda} \geq \delta_{\lambda, \varepsilon}-C \varepsilon-o(1) .
$$

Then by (3.55) and (6.6), we obtain

$$
\begin{aligned}
T F(2 n \pi)= & t_{2(n+1) \pi-\varepsilon, \lambda} F(2(n+1) \pi) \\
& +\left\{T-t_{2(n+1) \pi-\varepsilon, \lambda}-(2 n-1) \delta_{\lambda, \varepsilon}\right\} F(2 n \pi) \\
& +2 \delta_{\lambda, \varepsilon} \sum_{k=0}^{n-1} F(2 k \pi)+O(\varepsilon)+o(1) \\
\geq & T F(2 n \pi)+t_{2(n+1) \pi-\varepsilon, \lambda}\{F(2(n+1) \pi)-F(2 n \pi)\}
\end{aligned}
$$




$$
\begin{aligned}
& -\delta_{\lambda, \varepsilon} F(2 n \pi)-2 \delta_{\lambda, \varepsilon} \sum_{k=1}^{n-1}(F(2 n \pi)-F(2 k \pi))-C \varepsilon-o(1) \\
\geq & T F(2 n \pi)+t_{2(n+1) \pi-\varepsilon, \lambda} H(n)-C \varepsilon-o(1) .
\end{aligned}
$$

By (A.5.n), (6.6) and (6.7), for $\lambda \gg 1$, we obtain

$$
t_{2(n+1) \pi-\varepsilon, \lambda} \leq C \varepsilon+o(1), \quad \delta_{\lambda, \varepsilon} \leq C \varepsilon+o(1) .
$$

Since we assume $\left\|u_{\lambda}\right\|_{\infty} \geq 2(n+1) \pi$ for $\lambda \gg 1$, by the argument of Lemmas 2.7, 3.6, 3.9 and 3.10, we have (5.7). Then it follows from (3.55) for $1 \leq k \leq n,(3.57)$ and (6.8) that

$$
t_{2(n+1) \pi-\varepsilon, \lambda}=T-(2 n+1) \delta_{\lambda, \varepsilon}-O(\varepsilon)-o(1) \geq T-C \varepsilon-o(1) .
$$

This implies that

$$
T F(2 n \pi) \geq t_{2(n+1) \pi-\varepsilon, \lambda} F(2(n+1) \pi-\varepsilon) \geq T F(2(n+1) \pi)-C \varepsilon-o(1) .
$$

This is a contradiction. Hence we obtain $\left\|u_{\lambda}\right\|_{\infty}<2(n+1) \pi$ for $\lambda \gg 1$. Then by this and (4.1), we have

$$
\lambda\left(1-\cos \left\|u_{\lambda}\right\|_{\infty}\right) \leq \mu(\lambda) F(2(n+1) \pi) .
$$

Since $u_{\lambda} \in M_{2 n \pi}$, by this and Lemma 2.4, we have only two possibilities: $\left\|u_{\lambda}\right\|_{\infty} \rightarrow 2(n+1) \pi$ or $\left\|u_{\lambda}\right\|_{\infty} \rightarrow 2 n \pi$ as $\lambda \rightarrow \infty$. Firstly, if $\left\|u_{\lambda}\right\|_{\infty} \rightarrow 2 n \pi$ as $\lambda \rightarrow \infty$, then $u_{\lambda} \rightarrow 2 n \pi$ locally uniformly on $(0, T)$ as $\lambda \rightarrow \infty$, since $u_{\lambda} \in M_{2 n \pi}$. So we obtain Theorem 4 . Secondly, consider the case where $\left\|u_{\lambda}\right\|_{\infty} \rightarrow 2(n+1) \pi$ as $\lambda \rightarrow \infty$. Then by the same argument as those of Lemma 3.12, we have (6.6) for $\lambda \gg 1$. By this and (3.55) for $1 \leq k \leq n-1$, we also obtain (6.7), which implies (6.8). Then by (3.55) for $1 \leq k \leq n-1$, (3.57) and (6.8), we obtain

$$
l_{\lambda, \varepsilon, n}+m_{\lambda, \varepsilon, n} \geq T-C \varepsilon-o(1) .
$$

This implies that $u_{\lambda} \rightarrow 2 n \pi$ locally uniformly on $(0, T)$ as $\lambda \rightarrow \infty$. Thus the proof is complete.

\section{Appendix}

In this section we prove Lemmas 2.3, 2.4 and Theorem 1(iii) for completeness.

Proof of Lemma 2.3. We put

$$
w_{\lambda}(t)= \begin{cases}-\lambda^{1 / 2}|t|+\lambda^{1 /(2(m+1))} & 0 \leq|t| \leq \lambda^{-m /(2(m+1))}, \\ 0 & \lambda^{-m /(2(m+1))}<|t| \leq T .\end{cases}
$$

For a fixed $\lambda>0$, we put $g(\gamma):=K\left(\gamma w_{\lambda}\right)$ for $\gamma \geq 0$. Then clearly, $g(0)=0$ and $g(\gamma) \rightarrow \infty$ as $\gamma \rightarrow \infty$. Hence there exists $c_{\lambda}>0$ such that $g\left(c_{\lambda}\right)=2 T F(\alpha)$. 
This implies $V_{\lambda}:=c_{\lambda} w_{\lambda} \in M_{\alpha}$. By (A.4), there exist constants $C_{3}, C_{4}>0$ such that for $s \geq 0$

$$
F(s) \geq C_{3} s^{m}-C_{4}
$$

By this, we have

$2 T F(\alpha)=K\left(V_{\lambda}\right)=\int_{I} F\left(V_{\lambda}(t)\right) d t \geq \int_{I}\left(C_{3} V_{\lambda}(t)^{m}-C_{4}\right) d s=\frac{2 C_{3}}{m+1} c_{\lambda}^{m}-2 C_{4} T$.

This implies that $c_{\lambda} \leq C$ for $\lambda \gg 1$. Then by direct calculation, we obtain

$$
\left\|V_{\lambda}^{\prime}\right\|_{2}^{2}=2 c_{\lambda}^{2} \lambda^{(m+2) / 2(m+1)}, \quad \lambda \int_{I}\left(1-\cos V_{\lambda}(t)\right) d t \leq 4 \lambda^{(m+2) / 2(m+1)} .
$$

By this, we obtain our conclusion, since $\beta(\lambda)=L_{\lambda}\left(u_{\lambda}\right) \leq L_{\lambda}\left(V_{\lambda}\right)$.

Proof of Lemma 2.4. By Lemma 2.3, we have

$$
\begin{aligned}
\left\|u_{\lambda}^{\prime}\right\|_{2}^{2} & \leq C \lambda^{(m+2) /(2(m+1))}, \\
\int_{I}\left(1-\cos u_{\lambda}(t)\right) d t & \leq C \lambda^{-m /(2(m+1))} .
\end{aligned}
$$

Multiply (1.1) by $u_{\lambda}$ and integrate it over $I$. Then integration by parts along with (A.4) yields

$$
\text { (7.4) } \begin{aligned}
2 m T F(\alpha) \mu(\lambda) & =\mu(\lambda) \int_{I} m F\left(u_{\lambda}(t)\right) d t \leq \mu(\lambda) \int_{I} f\left(u_{\lambda}(t)\right) u_{\lambda}(t) d t \\
& =\left\|u_{\lambda}^{\prime}\right\|_{2}^{2}+\lambda \int_{I} u_{\lambda}(t) \sin u_{\lambda}(t) d t .
\end{aligned}
$$

To obtain our conclusion, we estimate $\int_{I} u_{\lambda}(t) \sin u_{\lambda}(t) d t$. By (7.1), we have

$$
\int_{I} u_{\lambda}(t)^{m} d t \leq \frac{1}{C_{3}}\left\{\int_{I} F\left(u_{\lambda}(t)\right) d t+2 C_{4} T\right\}=C_{5}^{m}:=\frac{1}{C_{3}}\left(2 T F(\alpha)+2 C_{4} T\right) .
$$

By this and Hölder's inequality, we obtain

$$
\begin{aligned}
\xi_{\lambda} & :=\left|\int_{I} u_{\lambda}(t) \sin u_{\lambda}(t) d t\right| \\
& \leq\left(\int_{I}\left|\sin u_{\lambda}(t)\right|^{q} d t\right)^{1 / q}\left(\int_{I} u_{\lambda}(t)^{m} d t\right)^{1 / m} \\
& =C\left(\int_{I}\left|\sin u_{\lambda}(t)\right|^{q} d t\right)^{1 / q},
\end{aligned}
$$

where $1 / q+1 / m=1$. Let $0<\varepsilon \ll 1$ be fixed. Then

$$
2 T F(\alpha)=2 \int_{0}^{T} F\left(u_{\lambda}(t)\right) d t \geq 2 \int_{0}^{\varepsilon / 2} F\left(u_{\lambda}(t)\right) d t \geq \varepsilon F\left(u_{\lambda}(\varepsilon / 2)\right) .
$$

By (7.1) and (7.6), we see that $u_{\lambda}(\varepsilon / 2) \leq C_{\varepsilon}$ for $\lambda \gg 1$. We choose $k_{\varepsilon} \in \mathbb{N}$ such that $C_{\varepsilon}<2 k_{\varepsilon} \pi$. For $0<\delta \ll 1$ and $k \in \mathbb{N}$, we put $J_{\lambda, k, \delta}:=\{t \in I$ : 
$\left.2(k-1) \pi+\delta<u_{\lambda}(t)<2 k \pi-\delta\right\}$. We choose $\delta>0$ so small that $\left|\sin u_{\lambda}(t)\right|<\varepsilon / 2$ for $t \in(\varepsilon / 2, T) \backslash\left(\sum_{k=1}^{k_{\varepsilon}} J_{\lambda, k, \delta}\right)$. Then for $\lambda \gg 1$, by (2.7), we obtain

$$
\begin{aligned}
\int_{I}\left|\sin u_{\lambda}(t)\right|^{q} d t \leq & 2 \int_{0}^{T}\left|\sin u_{\lambda}(t)\right| d t=2 \int_{0}^{\varepsilon / 2}\left|\sin u_{\lambda}(t)\right| d t \\
& +2 \int_{(\varepsilon / 2, T) \backslash\left(\sum_{k=1}^{k_{\varepsilon}} J_{\lambda, k, \delta}\right)}\left|\sin u_{\lambda}(t)\right| d t \\
& +2 \int_{(\varepsilon / 2, T) \cap\left(\sum_{k=1}^{k_{\varepsilon}} J_{\lambda, k, \delta}\right)}\left|\sin u_{\lambda}(t)\right| d t \\
\leq & +T \varepsilon+\left|\sum_{k=1}^{k_{\varepsilon}} J_{\lambda, k, \delta}\right|<C \varepsilon .
\end{aligned}
$$

This along with (7.5) implies that $\xi_{\lambda} \rightarrow 0$ as $\lambda \rightarrow \infty$. From this, (7.2) and (7.4), our conclusion follows.

Proof of Theorem 1(iii). If $\left\|u_{\lambda}\right\|_{\infty} \geq 2 \pi$ for $\lambda \gg 1$, then the assertion follows from (3.38) and Theorem 1(ii). Assume that there exists a subsequence of $\left\{u_{\lambda}\right\}$, denoted by $\left\{u_{\lambda}\right\}$ again, such that $\left\|u_{\lambda}\right\|_{\infty}<2 \pi$. Let $J:=\left[t_{1}, t_{2}\right] \subset\left(0, T_{\alpha, 0}\right)$ be fixed $\left(t_{1}<t_{2}\right)$. We choose $0<\varepsilon \ll 1$ sufficiently small. Then $t_{2}<t_{2 \pi-\varepsilon, \lambda}$ for $\lambda \gg 1$. Note that $\sin u_{\lambda}(t)<0$ for $t \in\left[0, t_{2}\right]$. By the equation in (1.1) and Lemma 2.1, we see that $-u_{\lambda}^{\prime \prime}(t)=\mu(\lambda) f\left(u_{\lambda}(t)\right)-\lambda \sin u_{\lambda}(t)>0$. Hence $-u_{\lambda}^{\prime}(t)$ is increasing on $J$. Then

$$
\varepsilon \geq u_{\lambda}\left(t_{1}\right)-u_{\lambda}\left(t_{2}\right)=\int_{t_{1}}^{t_{2}}\left(-u_{\lambda}^{\prime}(t)\right) d t \geq\left(t_{2}-t_{1}\right)\left|u_{\lambda}^{\prime}\left(t_{1}\right)\right| .
$$

This implies that $\left|u_{\lambda}^{\prime}\left(t_{1}\right)\right| \leq C \varepsilon$ for $\lambda \gg 1$. Now, by this and the equation (1.1), for $\lambda \gg 1$, we obtain

$$
\begin{aligned}
C \varepsilon & \geq\left|u_{\lambda}^{\prime}\left(t_{1}\right)\right|=-u_{\lambda}^{\prime}\left(t_{1}\right)=\int_{0}^{t_{1}}-u_{\lambda}^{\prime \prime}(s) d s \\
& =\mu(\lambda) \int_{0}^{t_{1}} f\left(u_{\lambda}(t)\right) d t-\lambda \int_{0}^{t_{1}} \sin u_{\lambda}(t) d t \geq \mu(\lambda)\left(\min _{2 \pi-\varepsilon \leq u \leq 2 \pi} f(u)\right) t_{1} .
\end{aligned}
$$

Thus the proof is complete.

Acknowledgment. This work was done while the author was visiting Université catholique de Louvain. He is thankful to Professor J. Mawhin and the faculty there for warm hospitality he received during his stay.

\section{REFERENCES}

[1] A. I. Bobenko And S. B. Kuksin, The nonlinear Klein-Gordon equation on an interval as a perturbed Sine-Gordon equation, Comment. Math. Helv. 70 (1995), 63-112.

[2] B. Gidas, W. M. Ni And L. Nirenberg, Symmetry and related properties via the maximum principle, Comm. Math. Phys. 68 (1979), 209-243. 
[3] R. E. O'Malley, JR., Singular Perturbation Methods for Ordinary Differential Equations, Springer, 1991.

[4] T. Shibata, Asymptotic behavior of eigenvalues of two-parameter nonlinear SturmLiouville problems, J. Anal. Math. 66 (1995), 277-294.

[5] - Interior transition layers of solutions to perturbed Sine-Gordon equation on an interval (to appear).

[6] Spectral asymptotics and bifurcation for nonlinear multiparameter elliptic eigenvalue problems, Bull. Belg. Math. Soc. Simon Stevin 3 (1996), 501-515.

Manuscript received December 12, 1999

Tetsutaro Shibata

Division of Mathematical and Information Sciences

Faculty of Integrated Arts and Sciences

Hiroshima University

Higashi-Hiroshima, 739-8521, JAPAN

E-mail address: shibata@mis.hiroshima-u.ac.jp 